ARTICLE

\title{
Ligand-directed two-step labeling to quantify neuronal glutamate receptor trafficking
}

Kento Ojima1, Kazuki Shiraiwa1', Kyohei Soga², Tomohiro Doura², Mikiko Takato', Kazuhiro Komatsu', Michisuke Yuzaki (i) ${ }^{3}$, Itaru Hamachi (i) ${ }^{1 凶}$ \& Shigeki Kiyonaka (i) ${ }^{2 凶}$

The regulation of glutamate receptor localization is critical for development and synaptic plasticity in the central nervous system. Conventional biochemical and molecular biological approaches have been widely used to analyze glutamate receptor trafficking, especially for $\alpha$ amino-3-hydroxy-5-methyl-4-isoxazole-propionate-type glutamate receptors (AMPARs). However, conflicting findings have been reported because of a lack of useful tools for analyzing endogenous AMPARs. Here, we develop a method for the rapid and selective labeling of AMPARs with chemical probes, by combining affinity-based protein labeling and bioorthogonal click chemistry under physiological temperature in culture medium. This method allows us to quantify AMPAR distribution and trafficking, which reveals some unique features of AMPARs, such as a long lifetime and a rapid recycling in neurons. This method is also successfully expanded to selectively label $\mathrm{N}$-methyl-D-aspartate-type glutamate receptors. Thus, bioorthogonal two-step labeling may be a versatile tool for investigating the physiological and pathophysiological roles of glutamate receptors in neurons.

\footnotetext{
${ }^{1}$ Department of Synthetic Chemistry and Biological Chemistry, Graduate School of Engineering, Kyoto University, Kyoto 615-8510, Japan. ${ }^{2}$ Department of Biomolecular Engineering, Graduate School of Engineering, Nagoya University, Nagoya 464-8603, Japan. ${ }^{3}$ Department of Physiology, School of Medicine,

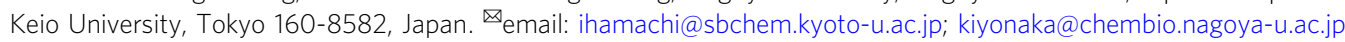


$\mathrm{n}$ the central nervous system, ionotropic glutamate receptors (iGluRs) mediate fast excitatory neurotransmission. iGluRs are categorized into distinct classes based on their pharmacology and structural homology, including the a-amino-3-hydroxy-5methyl-4-isoxazole-propionate (AMPA) receptor (GluA1-4), kainate receptor (GluK1-5), N-methyl-D-aspartate (NMDA) receptor (GluN1, GluN2A-D, GluN3A-B), and $\delta$ receptors $\left(\right.$ GluD1-2) ${ }^{1}$. iGluRs assemble as tetramers, and functional receptors are formed exclusively by the assembly of subunits within the same functional receptor class.

AMPA receptors (AMPARs), which are mainly permeable to monovalent cations $\left(\mathrm{Na}^{+}\right.$and $\left.\mathrm{K}^{+}\right)$, mediate the majority of excitatory synaptic transmission. AMPARs can form homotetramers or heterotetramers, and subunit compositions are dependent on brain regions. In hippocampal CA1 neurons, the majority of AMPARs are made up of GluA1/A2 and GluA2/ A3 subunit combinations, with a small contribution of GluA1 homomers ${ }^{2,3}$. Recent studies have revealed that AMPARs are constitutively cycled in and out of the postsynaptic membrane through endocytosis and exocytosis. The precise regulation of this process is critical for synaptic plasticity, which is the basis of learning, memory, and development in neural circuits ${ }^{2,3}$. Although AMPARs and kainate receptors are activated by glutamate binding, NMDA receptors (NMDARs), which have high permeability to $\mathrm{Ca}^{2+}$, require depolarization as well as agonist binding for their activation. Functional NMDARs require the assembly of two GluN1 subunits together with either two GluN2 subunits, or a combination of GluN2 and GluN3 subunits ${ }^{1}$. An NMDAR-dependent $\mathrm{Ca}^{2+}$ influx triggers intracellular signal transduction cascades, and the precise targeting of NMDARs to synapses is essential for controlling neuronal connectivity or neuroplasticity ${ }^{4}$. Thus, to understand the molecular mechanisms of learning and memory, it is critical to analyze the membrane localization and trafficking of iGluRs.

Biochemical approaches, such as surface biotinylation assays or related methods, have been widely used to analyze membrane protein localizations, and these methods have been successfully applied to AMPARs ${ }^{57}$. Although they are powerful tools for the analysis of AMPAR trafficking, cell-surface proteins are randomly labeled with biotin using these methods. As a result, purification of biotin-labeled AMPAR is required, which hampers quantitative analyses of trafficking. In contrast, to selectively visualize glutamate receptors, fluorescent proteins are fused to the receptors using genetically encoded approaches. For example, a $\mathrm{pH}$-sensitive variant of GFP (super-ecliptic pHluorin [SEP]) can be fused to the extracellular region of receptors to visualize cell-surface receptors in live neurons 8,9 . Instead of fluorescent proteins, protein tags such as SNAP- or Halo-tags are fused to the receptors for the covalent labeling of small chemical probes at the time that the probes are added $^{10-12}$. The downsizing of these protein tags has been successfully demonstrated by using a short peptide tag $(1-3 \mathrm{kDa})$ and its probe pair ${ }^{13-15}$. More recently, genetic code expansion in combination with bioorthogonal click chemistry has been reported for the fluorescent labeling of iGluRs in HEK293T cells, in which chemical probes are covalently attached to the side chain of an unnatural amino acid residue ${ }^{16,17}$. These genetically encoded approaches have been widely used in trafficking studies of iGluRs, especially for AMPARs. However, in most cases, these methods largely rely on the overexpression of target iGluR subunits. Given the formation of heterotetramers consisting of different subunits in iGluRs, the overexpression of a single iGluR subunit may interfere with the localization and/or trafficking of native iGluRs in neurons. Ideally, endogenously expressed iGluRs should be tagged with small chemical probes ${ }^{18,19}$.

In situ chemical protein labeling is ideal for analyzing native proteins in live cells. Affinity-based protein labeling is a powerful technique for the selective modification of target proteins ${ }^{20-26}$. As a traceless affinity-based labeling method for cell-surface proteins, our group has reported ligand-directed acyl imidazole (LDAI) chemistry ${ }^{24,25}$. With this technique, small chemical probes including fluorophores are covalently attached to nucleophilic amino acid residues located near the ligand-binding site. Recently, we have developed an AMPAR-selective LDAI reagent, termed "chemical AMPAR modification 2" (CAM2) reagents, which allows us to label chemical probes to AMPARs endogenously expressed in cultured neurons or acutely prepared brain slices $^{26}$. Although this technique is powerful for the selective modification of chemical probes to AMPARs, there are some restrictions for visualizing or analyzing cell-surface AMPARs. First, live cells need to be kept at low temperatures (e.g., $17^{\circ} \mathrm{C}$ ) during CAM2 labeling (1-4 h) to suppress the internalization of labeled AMPARs ${ }^{27}$. Second, the neuronal culture medium needs to be exchanged for serum-free medium or buffered saline during labeling to decrease non-specific labeling of serum proteins such as albumin. The relatively long-term exposure $(1-4 \mathrm{~h})$ to these non-physiological conditions may interfere with neuronal activity or survival ${ }^{28-30}$. Ideally, neurons should be kept under physiological conditions during chemical labeling.

Here, we show a method for the rapid and selective labeling of AMPARs under physiological temperature in culture medium by combining LDAI-based protein labeling and the inverse electron demand Diels-Alder (IEDDA) reaction, a form of fast click chemistry $^{31-33}$. This two-step labeling allows the quantitative analyses of distribution and/or trafficking of endogenous AMPARs from short to long periods in cultured neurons. In addition, we successfully apply this technique to chemically label and study the trafficking of endogenous NMDARs in neurons.

\section{Results}

Rapid labeling of surface AMPARs by bioorthogonal two-step labeling. We propose a bioorthogonal two-step labeling technique, which combines LDAI-based protein labeling with the bioorthogonal IEDDA reaction for the rapid and selective modification of chemical probes to cell-surface iGluRs. For the first step, a strained alkene is covalently attached to iGluRs using LDAI chemistry, where the acyl substitution reaction to nucleophilic amino acid residues is facilitated by selective ligand-protein recognition (first step in Fig. 1a). Next, the labeled alkene group is rapidly modified with tetrazine-conjugated probes (Tz-probes) on the cell surface, as a result of the high selectivity and high reaction rate of the IEDDA reaction (second step in Fig. 1a).

For the selective labeling of a strained alkene to AMPARs in the first step, we designed a CAM2 reagent bearing trans-cyclooctene (TCO), which we termed CAM2(TCO) (Fig. 1b). TCO was selected as the strained alkene because of its extremely fast cycloaddition kinetics in the IEDDA reaction. Compared with the original CAM2 reagents (e.g., CAM2(Ax488)) that bear aromatic fluorophores (see Supplementary Fig. 1a, b), an ethylene glycol linker is added between the reactive acyl imidazole unit and the TCO group in CAM2(TCO) to increase its hydrophilicity. Hydrophobic or aromatic groups have high affinity to albumin abundantly contained in serum ${ }^{34}$; therefore, this improvement decreases the undesired labeling of albumin, which allows the chemical labeling of AMPARs to be conducted in cell culture medium containing serum or substitutes. In addition, the first labeling is conducted at a physiological temperature $\left(37^{\circ} \mathrm{C}\right)$. Although some of the labeled AMPARs are likely to be internalized in this condition, this is not problematic with the two-step labeling technique. This is because the chemical probes are selectively tethered to cell-surface AMPARs in the second step reaction (Fig. 1d). 
a

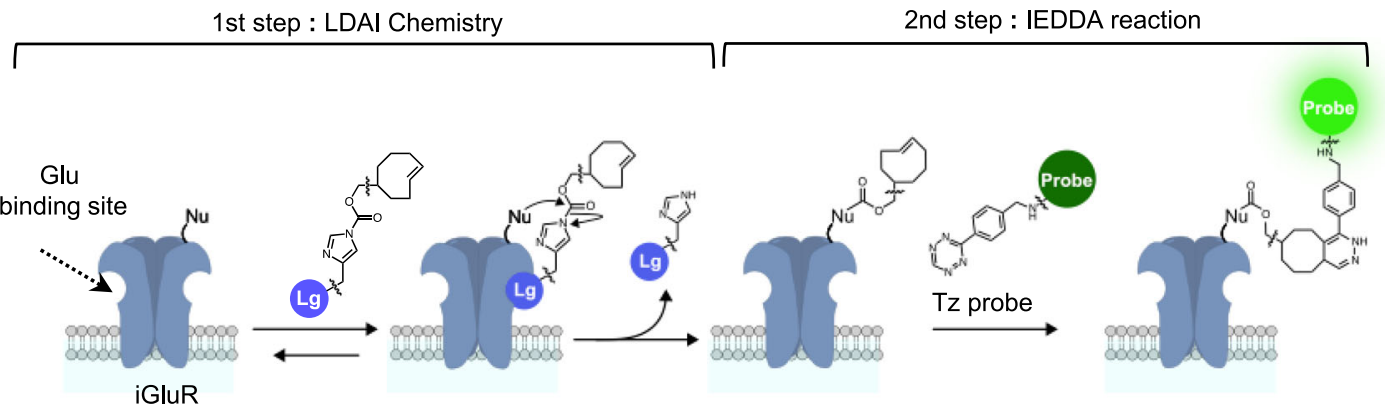

b<smiles>CC(C)(C)C1CC2CCC(CC2)C1</smiles>

CAM2(TCO)<smiles>CCC(=O)N(CC(=O)O)c1cccc(-n2ccc(CNC(=O)CCC(=O)NCCc3cn(C(=O)CCOCCNC(=O)CCOCCOCCOCCOCCNC(=O)OC4CC5CCC(CC5)C4)cn3)c2)c1</smiles>

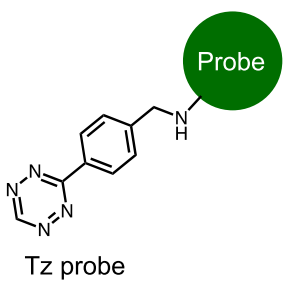

Reactive site

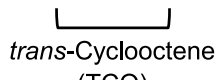
(PFQX)

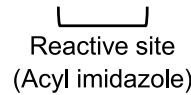

(TCO)
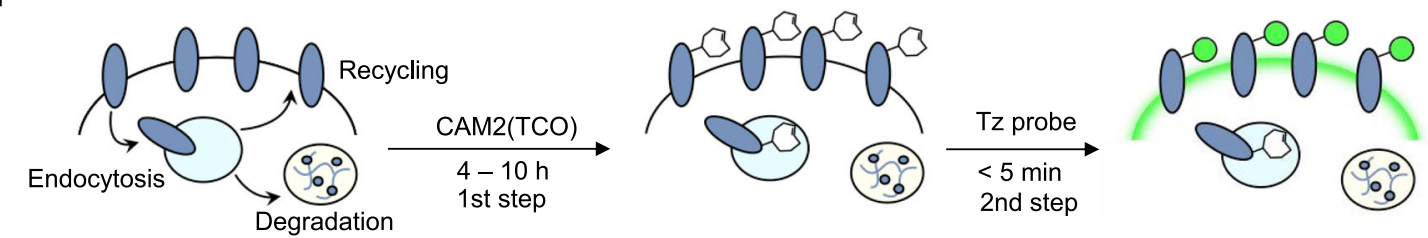

Fig. 1 Rapid labeling of cell-surface iGluRs by ligand-directed two-step labeling. a Schematic illustration of the two-step labeling to iGluRs. In the 1st step, a strained alkene is covalently attached to iGluRs by LDAI chemistry. In the 2nd step, Tz probe is selectively tethered by IEDDA reaction. Lg, selective ligand for iGluRs; Nu, nucleophilic amino acid residue. Glu, glutamate. b Chemical structure of CAM2(TCO). c Chemical structure of Tz probes. The detailed chemical structures are shown in Supplementary Fig. 2. d Schematic illustration of the two-step labeling in live cell.

Regarding the second step (the IEDDA reaction), the reaction rate is highly dependent on the chemical structure of the tetrazine group. We selected monoaryl tetrazine, which has both a fast reaction rate and high bioorthogonality, and prepared cellimpermeable Tz-probes bearing hydrophilic and anionic fluorophores or biotin for cell-surface labeling (Fig. 1c and Supplementary Fig. 2). Shortening the reaction time of the probe labeling not only contributes to cell-surface specific labeling, but also decreases the adsorption of the chemical probes to cells, culture dishes, or coverslips. Moreover, some tetrazinefluorophore conjugates have a "turn-on" response upon the IEDDA cycloaddition ${ }^{35-37}$, which contributes to a high signal-tonoise $(\mathrm{S} / \mathrm{N})$ ratio in fluorescence imaging.

Chemical labeling of surface AMPARs ectopically expressed in HEK293T cells. The designed two-step labeling method was initially examined in HEK293T cells transiently expressing GluA2, a main subunit of AMPARs. For the first step reaction, CAM2(TCO) was added to the culture medium, which included $10 \%$ fetal bovine serum (FBS), and the culture dish was incubated at $37^{\circ} \mathrm{C}$ for $4 \mathrm{~h}$. The second step reaction was performed for 5 min by adding membrane-impermeable $\mathrm{Tz}(\mathrm{Fl})$ for fluorescein labeling on the cell surface. As shown in Fig. 2a, western blotting of the cell lysate using anti-fluorescein (anti-Fl) antibodies showed a strong band around $110 \mathrm{kDa}$ (lane 1). This band was not observed in the cells co-treated with a competitive ligand (NBQX) or in any other control conditions (lanes 2-5). With regard to the molecular weight of the labeled band, the anti-Fl signal corresponded to the highest signal among multiple bands that were detected using anti-GluA2/3 antibodies (Fig. 2b). The multiple GluA2 bands converged into a single lower band after treatment with peptide- $N$-glycosidase F (PNGase F), which is consistent with previous reports showing that GluA2 is highly glycosylated with $\mathrm{N}$-linked sugars ${ }^{38}$. Importantly, in the PNGase F-treated samples, the shifted anti-GluA2 band merged with the anti-Fl signal (Fig. 2b). These findings indicate that highly glycosylated GluA2 is selectively labeled using our methods. Furthermore, in the case of direct fluorescein labeling using the original CAM2(Fl) under the same conditions (see Supplementary Fig. $1 \mathrm{~b}$ for its structure), there was a strong band around $70 \mathrm{kDa}$ as well as the $110 \mathrm{kDa}$ band (lanes 6-7 in Fig. 2a and Supplementary Fig. 3). The $70 \mathrm{kDa}$ band, whose intensity did not change even in the presence of NBQX, corresponds to albumin contained in serum (for details, see Fig. 2a legend). These results therefore indicate the high selectivity of the two-step labeling 
a

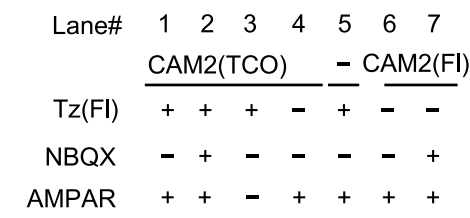

(kDa)
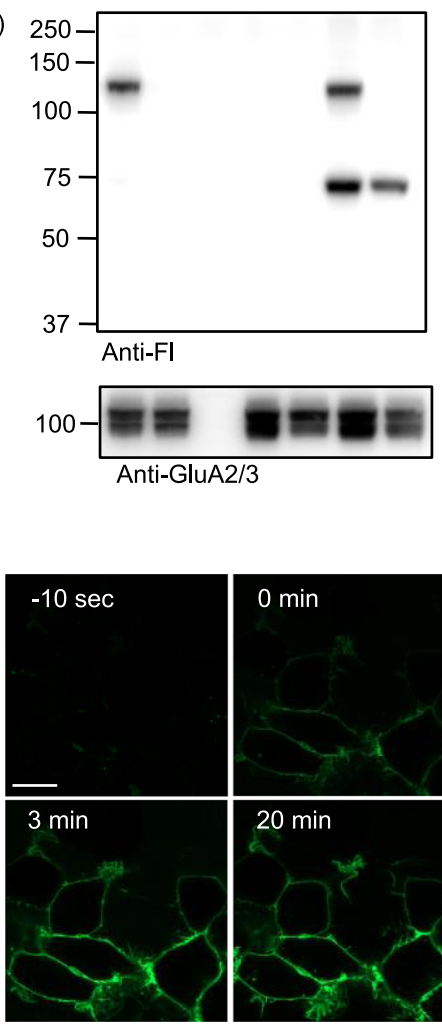

d

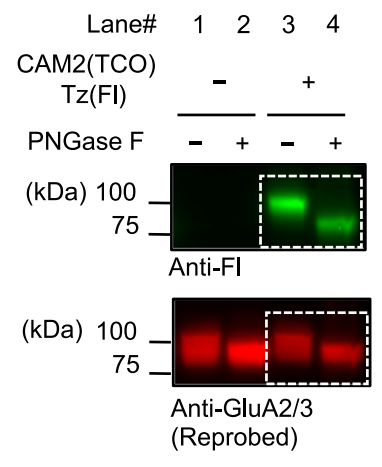

Lane\#

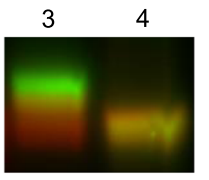

Overlay

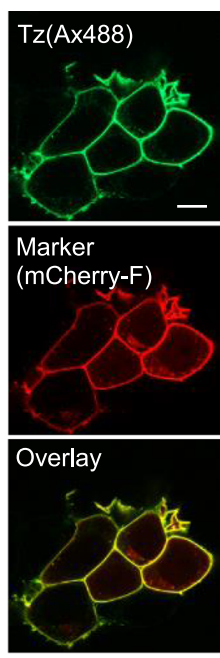

Fig. 2 The two-step labeling of cell-surface AMPARs ectopically expressed in HEK293T cells. a Western blotting analyses of HEK293T cells after the

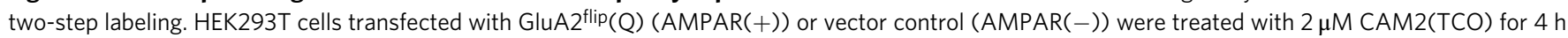

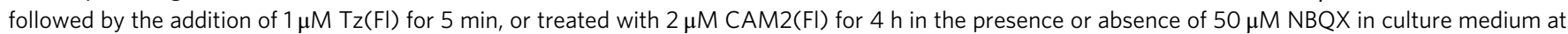
$37^{\circ} \mathrm{C}$. The cell lysates were analyzed by western blotting using anti-fluorescein or anti-GluA2/3 antibody. Quantification of the band intensity is shown in Supplementary Fig. 3. When CAM2(FI) is added in serum free medium, strong bands around $70 \mathrm{kDa}$ in lane \#6 and \#7 disappear (for details see ref. 26 ). b Effects of PNGase F treatment on the western blotting of labeled AMPAR in HEK293T cells expressing GluA2. Lower image shows the overlay of anti-FI image and anti-GluA2/3 image for lane \#3 and \#4. Two-step labeling was conducted as described in (a). PNGase F (1000 units/100 $\mu \mathrm{L}$ ) was added to the cell lysate. For details, see Methods section. c Confocal live imaging of the HEK293T cells labeled with $2 \mu$ M CAM2(TCO) and 0.1 $\mu$ M Tz(Ax488). Labeling was conducted as described in (a). mCherry- $F$ was utilized as a transfection marker. Scale bars, $10 \mu \mathrm{m}$. $\mathbf{d}$ Reaction kinetics of tetrazine ligation on live cells by confocal live imaging of the HEK293T cells labeled with $2 \mu \mathrm{M} \mathrm{CAM} 2(\mathrm{TCO})$ after addition of $0.3 \mu \mathrm{M}$ Tz(Ax488) at $37{ }^{\circ} \mathrm{C}$. In left, confocal images are shown. Scale bars, $20 \mu \mathrm{m}$. In right, time-course of the fluorescent intensity of Alexa 488 is shown ( $n=6$ cells). Data are represented as mean \pm s.e.m.

technique using CAM2(TCO) compared with the original CAM2 (Fl) under cell culture conditions.

For visualizing fluorescently labeled AMPARs on the cell surface, confocal microscopic live imaging was performed after the two-step labeling process under cell culture conditions. Here, $\mathrm{Tz}(\mathrm{Ax} 488)$ was used in the second step of labeling. Alexa 488 has bright fluorescence that is unaffected under endosomal acidic conditions; in contrast, fluorescein has weakened fluorescence under acidic conditions. Thus, Alexa 488 is more suitable to quantify the cellular distribution or trafficking of labeled AMPARs using fluorescent imaging. As shown in Fig. 2c, prominent fluorescence was observed exclusively from the cell surface in cells co-transfected with mCherry-F, a membranetargeted transfection marker. In contrast, fluorescent signals were not observed in control conditions, such as in CAM2(TCO)untreated or NBQX-co-treated cells (Supplementary Fig. 4). In the case of direct Alexa 488 labeling using CAM2(Ax488) in the same cell culture conditions, labeled signals were observed not only from the cell surface but also from the intracellular space (Supplementary Fig. 1c). This suggests that the two-step labeling technique is superior for the fluorescent visualization of cellsurface AMPARs under cell culture conditions. We also determined the reaction kinetics of rapid fluorophore labeling of cell-surface AMPARs with the help of the turn-on fluorescent property of $\mathrm{Tz}(\mathrm{Ax} 488)$ upon the IEDDA reaction (Supplementary Fig. 5). Immediately after adding $\mathrm{Tz}(\mathrm{Ax} 488)$, prominent fluorescent signals were observed from the cells co-transfected with the transfection marker mCherry-F (Fig. 2d), and the fluorescent signals were saturated within $3 \mathrm{~min}$. Thus, cell-surface AMPARs can be labeled by the fluorophore with fast kinetics.

By taking advantage of the high bioorthogonality of the IEDDA reaction, cell-surface AMPARs were successfully labeled with various kinds of chemical probes, ranging from small molecules to middle-sized molecules such as SeTau-647, a squaraine 
a

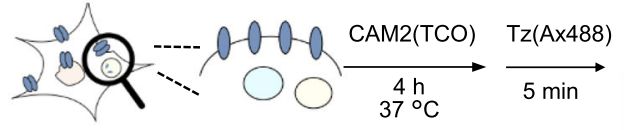
HEK293T

b

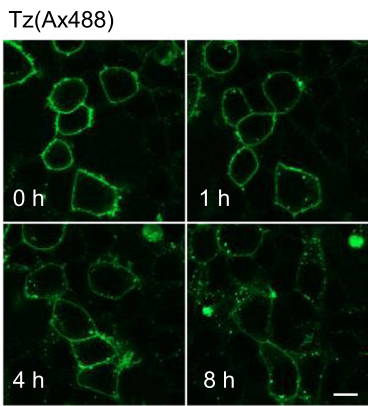

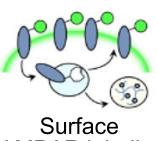
Surface
AMPAR labeling

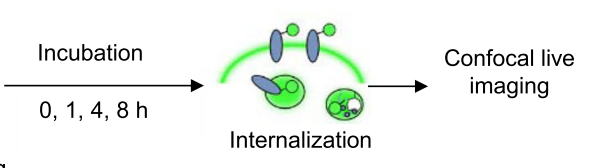

$0,1,4,8 \mathrm{~h}$

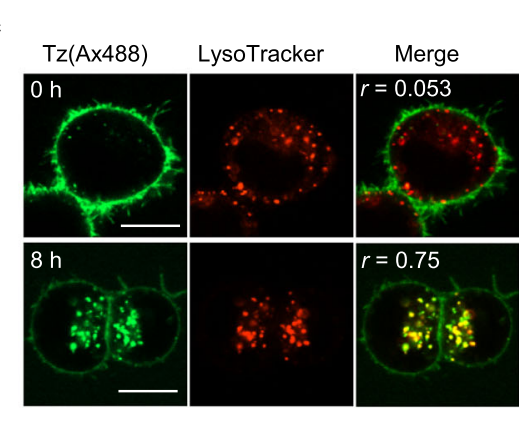

d

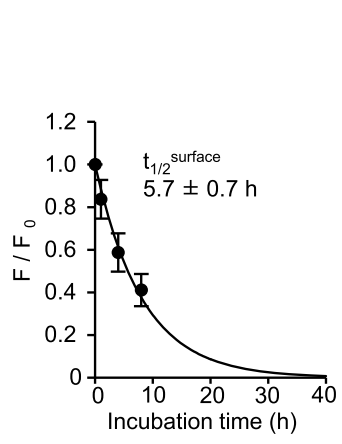

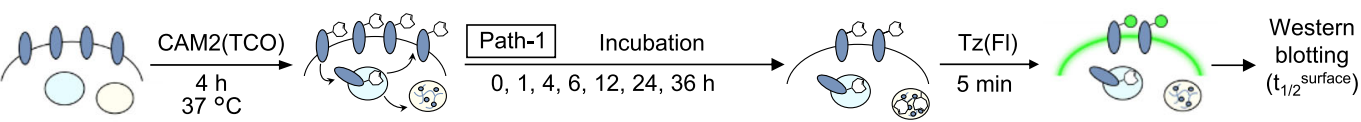

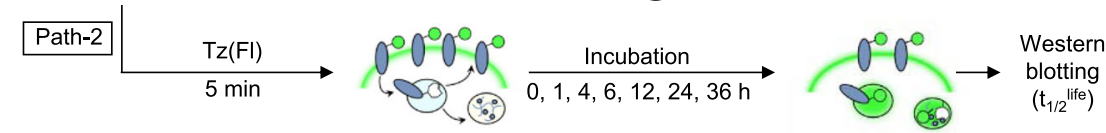

e
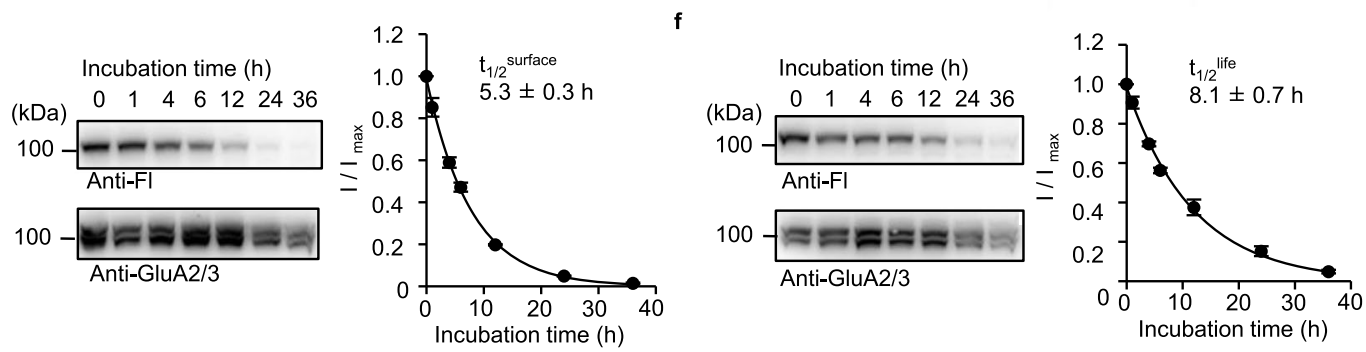

g

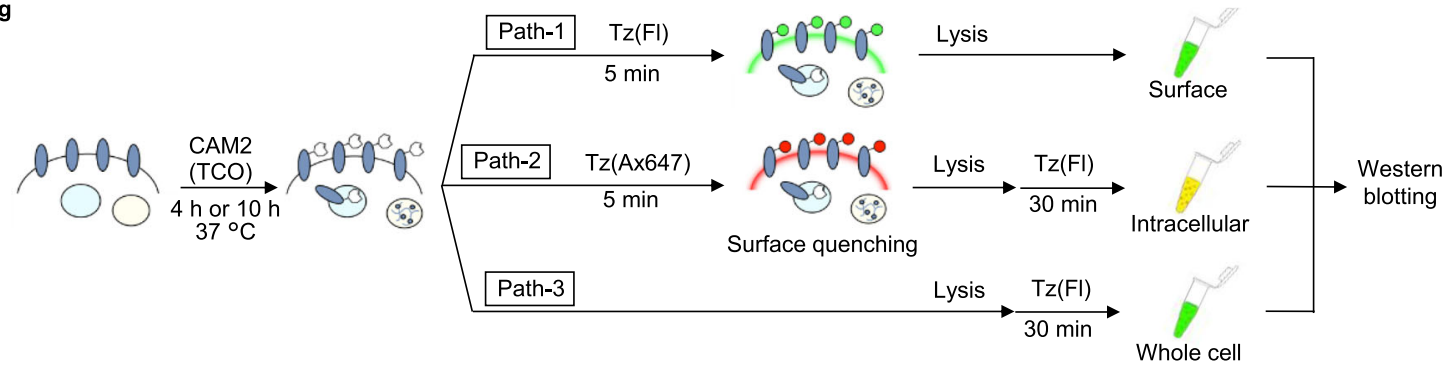

h TCO-AMPAR $(4 \mathrm{~h})$

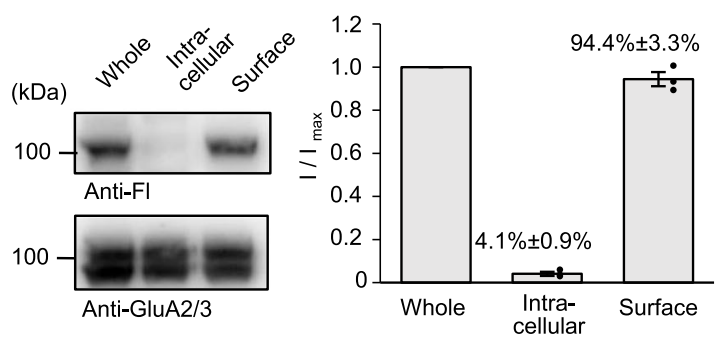

rotaxane dye that has high photostability and a long fluorescence lifetime ${ }^{39,40}$ (Supplementary Figs. 2 and 6). This probe flexibility is another feature of the two-step labeling technique that is superior compared with the direct labeling of probes using original CAM2 reagents, where each probe-tethered CAM2 needs to be synthesized (Supplementary Fig. 1b).

With regard to labeling efficacy, quantification of the remaining unlabeled GluA2 fraction showed that $35 \pm 3 \%$ of 


\section{Fig. 3 Quantitative analyses of AMPAR trafficking in HEK293T cells using the two-step labeling under the physiological cell culture condition.}

a Schematic illustration of trafficking analyses of cell-surface AMPARs by confocal microscopy. b Time-lapse confocal imaging of HEK293T cells after twostep labeling using CAM2(TCO) and Tz(Ax488). The HEK293T cells were transfected with GluA2 flip $(\mathrm{Q})$. In left, confocal images are shown. Scale bars, $10 \mu \mathrm{m}$. In right, time-course of the fluorescent intensity from the cell surface is shown ( $n=3$ biological replicates). [CAM2(TCO)] $=2 \mu \mathrm{M}$,

$\left[\mathrm{Tz}(\mathrm{Ax}\right.$ 488) $]=0.1 \mu \mathrm{M}$. c Co-staining of labeled AMPARs with LysoTracker ${ }^{\mathrm{TM}}$. The labeled HEK293T cells were incubated with LysoTracker ${ }^{\mathrm{TM}}$ Red dnd99 immediately after $\mathrm{Tz}(\mathrm{Ax} 488)$ labeling (upper panel) or subsequent $8 \mathrm{~h}$ incubation at $37^{\circ} \mathrm{C}$ (lower panel), and confocal live imaging was performed. Pearson's correlation coefficients $(r)$ are shown in the image. Scale bars, $10 \mu \mathrm{m}$. d-f Determination $t_{1 / 2}$ surface or $t_{1 / 2}$ life of AMPARs by western blotting. d Schematic illustration of the procedure is shown. In $\mathbf{e}$ and $\mathbf{f}, t_{1 / 2}$ surface and $t_{1 / 2}$ life are determined, respectively. In left, representative results of western blotting are shown. In right, time-course of the labeled band is shown $(n=3$ biological replicates). $[\mathrm{CAM} 2(\mathrm{TCO})]=2 \mu \mathrm{M},[\mathrm{Tz}(\mathrm{FI})]=1 \mu \mathrm{M}$.

$\mathbf{g}$, $\mathbf{h}$ Determination of TCO-labeled AMPARs on cell-surface, in intracellular area, or in whole-cell by western blotting. In $\mathbf{g}$, schematic illustration of the procedure is shown. In $\mathbf{h}$, intracellular and surface ratio after CAM2(TCO) labeling for $4 \mathrm{~h}$ are determined. In left, representative results of western blotting are shown. In right, band intensities for cell-surface and intracellular labeling were analyzed, both of which were normalized by that for whole-cell labeling $(n=3$ biological replicates). $[\mathrm{CAM} 2(\mathrm{TCO})]=2 \mu \mathrm{M},[\mathrm{Tz}(\mathrm{FI})]=1 \mu \mathrm{M}$. Data are represented as mean \pm s.e.m.

surface AMPARs were visualized in the two-step labeling (Supplementary Fig. 7). Moreover, AMPAR function was not visibly affected by the two-step labeling (Supplementary Fig. 8), which is consistent with our previous analyses that showed minimal disturbance of AMPAR ion channel properties by CAM2 labeling ${ }^{26}$.

Analyses of AMPAR trafficking in HEK293T cells. Once we had a potential labeling method for cell-surface AMPARs under cell culture conditions, we analyzed receptor trafficking using both live imaging and biochemical approaches. First, we analyzed cellsurface AMPAR trafficking in HEK293T cells using confocal live imaging. After incubating the cells with CAM2(TCO) under physiological temperature in culture medium, $\mathrm{Tz}(\mathrm{Ax} 488)$ was added to the culture medium to selectively visualize cell-surface AMPARs and cells were incubated for each period $(0-8 \mathrm{~h})$ (Fig. 3a). As shown in Fig. 3b, the labeled fluorescence on the cell surface decreased after incubation at $37^{\circ} \mathrm{C}$. Fluorescent granules were instead observed in the intracellular area, and most of the fluorescent signals were from intracellular granules after $8 \mathrm{~h}$ of incubation. The half-time of cell-surface AMPARs $\left(t_{1 / 2}\right.$ surface $)$, which includes both the remaining and recycled fractions, was calculated to be $5.7 \pm 0.7 \mathrm{~h}$ from the fluorescent intensity on the cell surface (Fig. $3 b$ ). In addition, the intracellular punctate signals merged with a fluorescent lysosome marker (LysoTracker) after $8 \mathrm{~h}$ of incubation, suggesting that internalized AMPARs were transported to lysosomes (Fig. 3c). Similar internalization behavior was observed when AMPARs were labeled with different fluorophores using $\mathrm{Tz}(\mathrm{Ax} 647)$ or $\mathrm{Tz}(\mathrm{ST} 647)$ (Supplementary Fig. 9).

Quantitative analyses of the fates of cell-surface AMPARs were examined using biochemical approaches. To quantify the $t_{1 / 2}$ surface of AMPARs, HEK293T cells were incubated for each period $(0-36 \mathrm{~h})$ after treatment with CAM2(TCO) (path-1 in Fig. 3d). Next, $\mathrm{Tz}(\mathrm{Fl})$ was added for the selective modification of fluorescein to cell-surface AMPARs. Using western blotting of the cell lysate, the $t_{1 / 2}$ surface of AMPARs was determined to be $5.3 \pm$ $0.3 \mathrm{~h}$ (Fig. 3e), which was similar to the value that was determined using confocal imaging (Fig. 3b). The half-time of the degradation $\left(t_{1 / 2}\right.$ life $)$ of cell-surface receptors was evaluated by modifying the protocols, where $\mathrm{Tz}(\mathrm{Fl})$ was added after CAM2(TCO) labeling (path-2 in Fig. 3d). The cells were then incubated for each period $(0-36 \mathrm{~h})$, and the cell lysates were subjected to western blotting. As shown in Fig. $3 \mathrm{f}, t_{1 / 2}$ life was determined to be $8.1 \pm 0.7 \mathrm{~h}$, which was slightly longer than the $t_{1 / 2}$ surface of cell-surface AMPARs $(p<0.05)$. Considering the colocalization of internalized AMPARs and lysosomes (Fig. 3c), the internalized AMPARs are likely decomposed via lysosomal degradation in HEK293T cells.

We next determined the intracellular versus surface percentages of TCO-labeled AMPARs (TCO-AMPARs) in each period, which can provide valuable information regarding the fate of cellsurface AMPARs. To quantify these percentages, cell-surface AMPARs in HEK293T cells treated with CAM2(TCO) were selectively labeled with fluorescein by adding cell-impermeable $\mathrm{Tz}$ (Fl) to the medium under live cell conditions (path-1 in Fig. 3g). To label intracellular TCO-AMPARs, surface TCO-AMPARs were first masked with cell-impermeable $\mathrm{Tz}(\mathrm{Ax} 647)$ (path-2 in Fig. 3g). After lysis of the cells, $\mathrm{Tz}(\mathrm{Fl})$ was added to the cell lysate to label intracellular TCO-AMPARs. The whole-cell-labeling fraction, where both cell-surface and intracellular TCO-AMPARs were labeled with fluorescein, was prepared by adding $\mathrm{Tz}(\mathrm{Fl})$ after cell lysis (path-3 in Fig. 3g). Prior to these analyses, we first investigated whether the second step reaction using $\mathrm{Tz}(\mathrm{Fl})$ proceeds rapidly and/or selectively in cell lysate. Western blotting analyses revealed that the covalent modification of fluorescein was selective to AMPARs in cell lysate (Supplementary Fig. 10), and the reaction was saturated after $15 \mathrm{~min}$ when either 0.1 or 0.3 $\mu \mathrm{M} \mathrm{Tz}(\mathrm{Fl})$ was added. The intracellular and cell-surface percentages of TCO-AMPARs after $4 \mathrm{~h}$ of incubation with CAM2(TCO) were analyzed using this protocol and determined to be $4.1 \pm 0.9 \%$ and $94.4 \pm 3.3 \%$, respectively (Fig. $3 \mathrm{~h}$ ), indicating that intracellular TCO-AMPAR levels were quite low.

Rapid labeling of endogenous AMPARs in neurons. We next examined the applicability of the bioorthogonal two-step labeling technique for the rapid modification of cell-surface AMPARs that are endogenously expressed in neurons. Primary cultured neurons from the cerebral cortex were incubated with CAM2(TCO) for $10 \mathrm{~h}$ under neuronal culture conditions, and $\mathrm{Tz}(\mathrm{Fl})$ was then added for $5 \mathrm{~min}$ for cell-surface labeling. Western blotting analyses of the cell lysate showed a single strong band corresponding to the molecular weight of AMPARs (see lane 1 in Fig. 4a). This band was not detected in the co-presence of the competitive ligand NBQX, or in other control conditions (see lanes 2-4 in Fig. 4a). As observed with the AMPARs expressed in HEK293T cells (Fig. 2a), smeared bands were detected using antiGluA2 antibodies; the anti-Fl band corresponded to the highest band in the smeared anti-GluA2 signals. After the removal of $\mathrm{N}$ linked sugars by PNGase treatment, the smeared anti-GluA2 bands converged into a single lower band, which merged with the anti-Fl signal (Supplementary Fig. 11). These results suggest that the highly glycosylated fraction of endogenous AMPARs were selectively labeled with fluorescein by the rapid labeling.

Of the AMPAR subunits (GluA1-4), GluA1, GluA2, and GluA3 are highly expressed in cultured cortical neurons ${ }^{41}$. We next examined the efficacy of our methods for visualizing tetrameric AMPARs by quantifying the remaining unlabeled GluA2 fraction. As shown in Supplementary Fig. 12a, $44 \pm 4 \%$ of GluA2-containing AMPARs were recognized by the two-step labeling method. Similarly, we calculated that $37 \pm 7 \%$ of GluA1- 
a

b

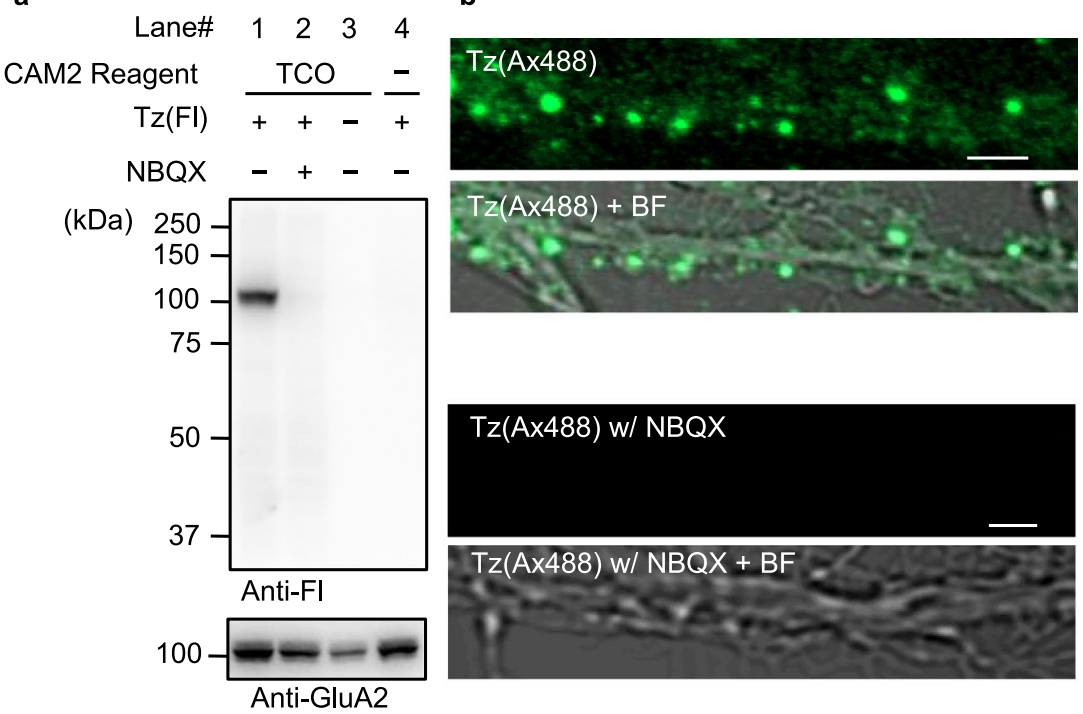

C
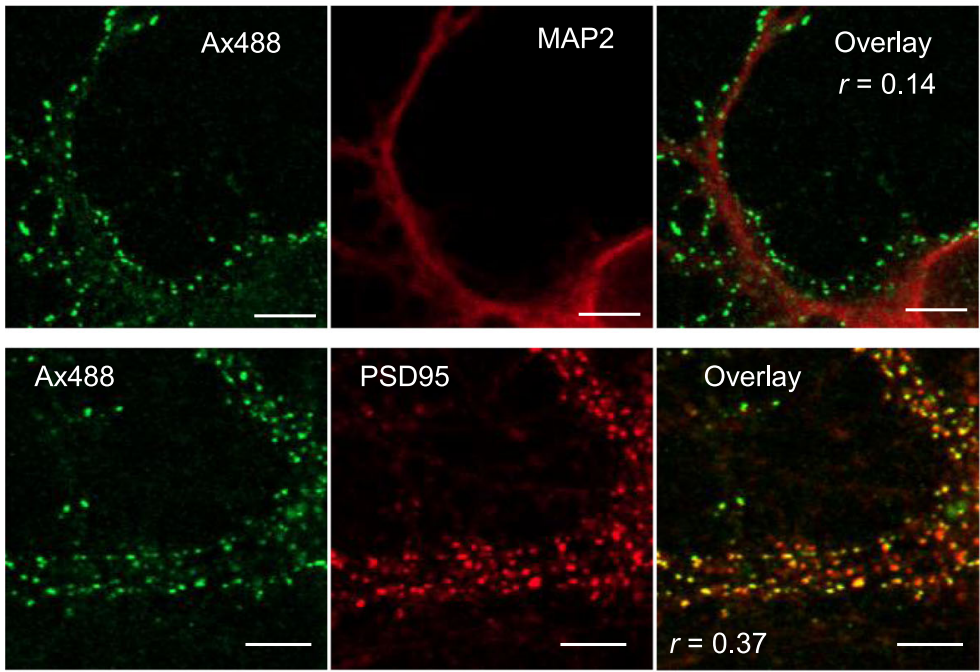

d
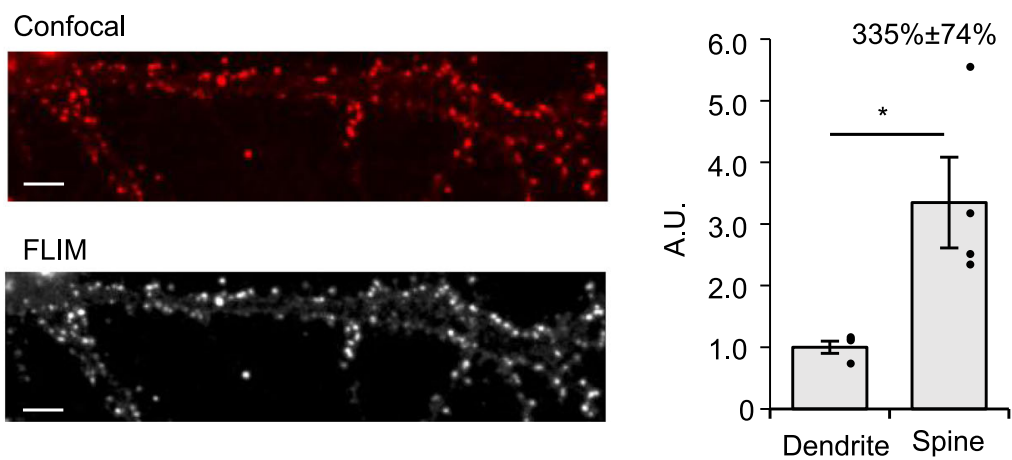

Fig. 4 The two-step labeling of cell-surface AMPARs endogenously expressed in neurons. a Western blotting analyses of cortical neurons after the twostep labeling. Primary cultured cortical neurons were treated with $2 \mu \mathrm{M} \mathrm{CAM} 2(\mathrm{TCO})$ for $10 \mathrm{~h}$ followed by the addition of $1 \mu \mathrm{M}$ Tz(FI) for $5 \mathrm{~min}$ in the presence or absence of $50 \mu \mathrm{M} \mathrm{NBQX}$ in culture medium at $37^{\circ} \mathrm{C}$. The cell lysates were analyzed by western blotting using anti-fluorescein or anti-GluA2 antibody. $\mathbf{b}$ Confocal live imaging of the neurons labeled with $2 \mu \mathrm{M} \mathrm{CAM} 2(\mathrm{TCO})$ and $0.1 \mu \mathrm{M} \mathrm{Tz}(\mathrm{Ax} 488)$. Labeling was conducted as described in a. Scale bars, $2 \mu \mathrm{m}$. c Immunostaining of cortical neurons after the two-step labeling. Labeling was conducted as described in $\mathbf{b}$. The neurons were fixed, permeabilized, and immunostained using anti-MAP2 (upper) or anti-PSD95 antibody (lower). Scale bars, $5 \mu$ m. Pearson's correlation coefficients ( $r$ ) are shown in the image. Whole images are shown in Supplementary Fig. 14a. d FLIM imaging and analyses of cell-surface AMPARs in the neurons after the two-step labeling. The neurons were prepared as described in c. In left, representative confocal image (upper) and FLIM image (lower) for a lifetime fraction $(\tau=2.4 \pm 0.1 \mathrm{~ns})$ are shown. Scale bars, $5 \mu \mathrm{m}$. In right, FLIM intensities in spine and dendrite were analyzed $(n=4 \mathrm{cells})$. [CAM2(TCO)] $=2 \mu \mathrm{M}$, $[\mathrm{Tz}(\mathrm{Ax} 488)]=0.1 \mu \mathrm{M}$. ${ }^{*}$ Significant difference $(p<0.05$ by two-sided Student's $t$-test. $p=0.048)$. Data are represented as mean \pm s.e.m. 
a

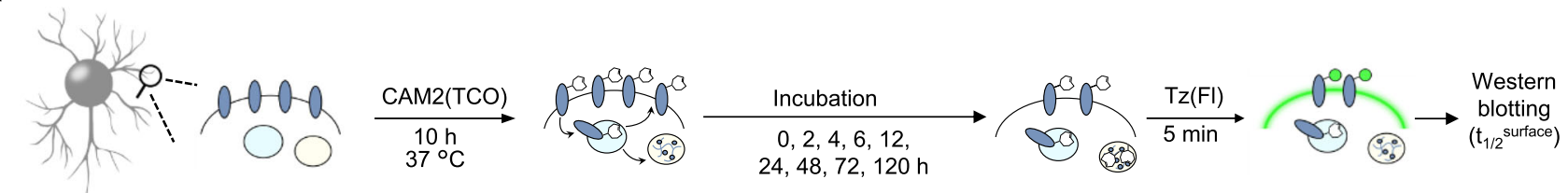

Cultured neuron

b

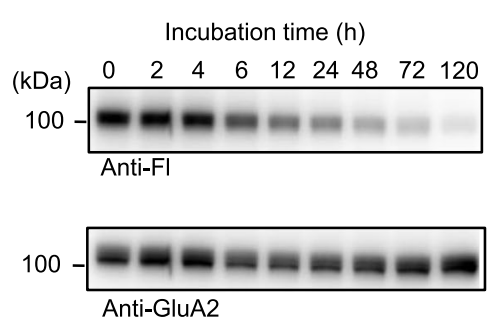

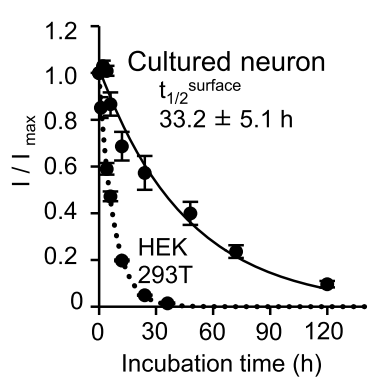

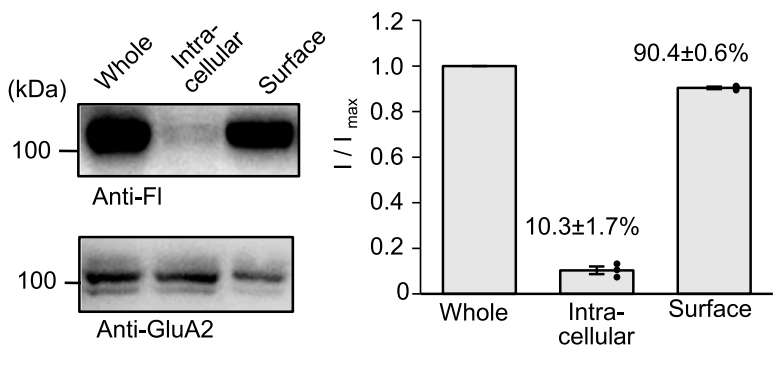

d

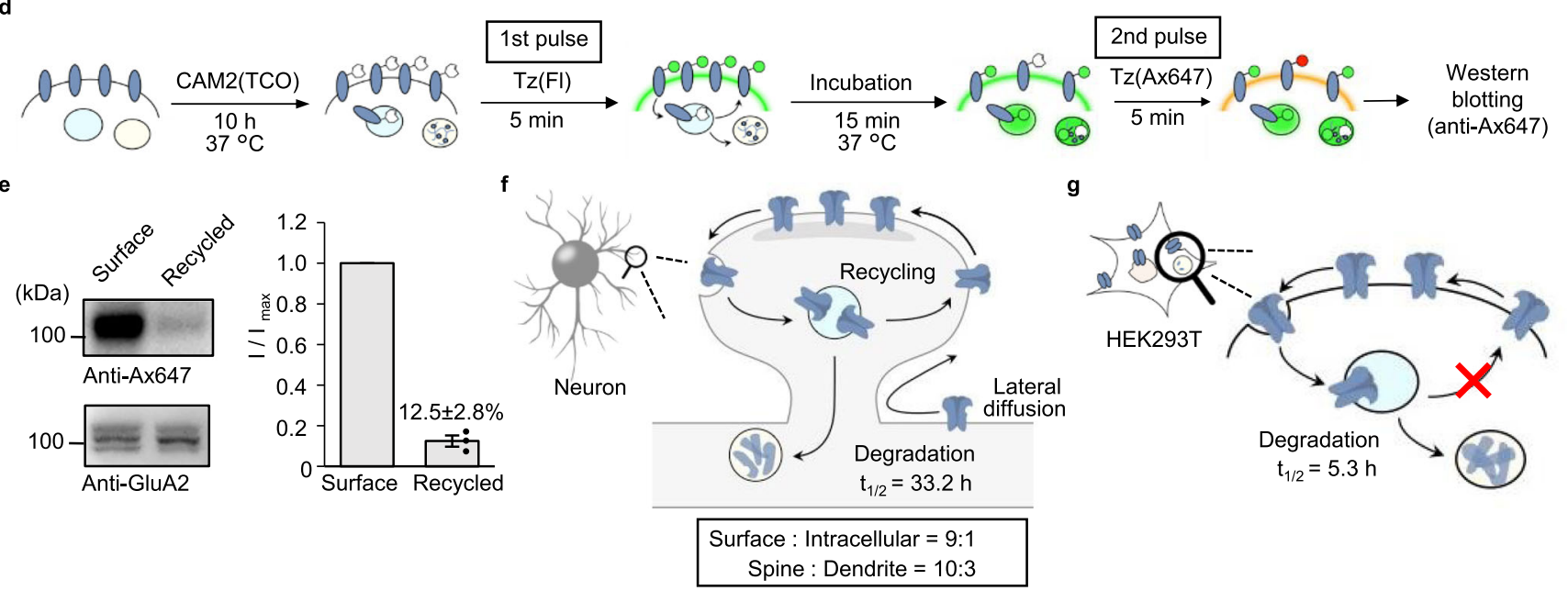

Fig. 5 Quantitative analyses of AMPAR trafficking in neurons using the two-step labeling under the physiological cell culture condition. a Schematic illustration of the procedure for determining $t_{1 / 2}$ surface for AMPARs. b Determination of $t_{1 / 2}$ surface by western blotting. In left, representative results of western blotting are shown. In right, time-course of the labeled band is shown ( $n=3$ biological replicates). [CAM2(TCO)] $=2 \mu \mathrm{M},[\mathrm{Tz}(\mathrm{FI})]=1 \mu \mathrm{M}$. c Determination of intracellular and surface ratio after CAM2(TCO) labeling for $10 \mathrm{~h}$. In left, representative results of western blotting are shown. In right, band intensities for cell-surface and intracellular labeling were analyzed, both of which were normalized by that for whole-cell labeling ( $n=3$ biological replicates). See also Supplementary Fig. 19 for tetrazine ligation in serum containing medium or in cell lysate. $[\mathrm{CAM} 2(\mathrm{TCO})]=2 \mu \mathrm{M},[\mathrm{Tz}(\mathrm{FI})]=1 \mu \mathrm{M}$. d, e Analyses of recycled AMPARs by pulse-chase-type analyses using the two-step labeling in neurons. In d, schematic illustration of the procedure is shown. In e, recycled AMPARs were analyzed by western blotting. In left, representative results of western blotting are shown. In right, exocytose AMPARs were quantified, which were normalized by that for surface labeling $(n=3$ biological replicates). $[\mathrm{CAM} 2(\mathrm{TCO})]=2 \mu \mathrm{M},[\mathrm{Tz}(\mathrm{FI})$ or $\mathrm{Tz}(\mathrm{Ax} 647)]=1 \mu \mathrm{M}$. Data are represented as mean \pm s.e.m. $\mathbf{f}, \mathbf{g}$ Trafficking and distribution of cell-surface AMPARs quantified by the two-step labeling in neuron (in $\mathbf{f}$ ) and HEK293T cells (in $\mathbf{g}$ ). Data are represented as mean \pm s.e.m.

and $43 \pm 5 \%$ of GluA3-containing AMPARs were recognized. However, considering the heterotetrameric formation of AMPAR subunits, we also needed to examine whether each subunit was covalently labeled with the probe or not. In this context, the immunoprecipitation assay in the denatured condition revealed that GluA2 and GluA3, but not GluA1, were covalently labeled with CAM2(TCO) (Supplementary Fig. 12b). This selectivity is consistent with our previous results ${ }^{26}$ in HEK293T cells. With regard to the efficacy of CAM2(TCO) labeling, the time-course of the labeling clearly indicated that chemical labeling occurred more efficiently at $37^{\circ} \mathrm{C}$ than in the previous condition at $17^{\circ} \mathrm{C}$ (Supplementary Fig. 13a). In addition, the concentration dependency of CAM2(TCO) revealed the $\mathrm{EC}_{50}$ value $(0.90 \pm 0.10 \mu \mathrm{M})$ of two-step labeling at $37^{\circ} \mathrm{C}$ in neurons (Supplementary Fig. 13b).
Using the two-step labeling technique in primary hippocampal neurons, fluorescently labeled AMPARs were visualized by confocal microscopy. At $5 \mathrm{~min}$ after the addition of $\mathrm{Tz}(\mathrm{Ax} 488)$, confocal live imaging showed punctate fluorescent signals from the CAM2(TCO)-treated neurons, and these signals were not observed in neurons co-treated with NBQX (Fig. 4b). To characterize the fluorescent signals in detail, $\mathrm{Tz}(\mathrm{Ax} 488)$-treated neurons were fixed with paraformaldehyde (PFA) and immunostained with anti-MAP2 or anti-PSD95 antibodies for dendritic or postsynaptic staining, respectively. As shown in Fig. 4c and Supplementary Fig. 14a, labeled Alexa 488 signals were observed alongside the anti-MAP2 signals, and merged well with the antiPSD95 signals. Considering the short incubation time with $\mathrm{Tz}$ (Ax488), the Alexa 488 signal likely corresponds to cell-surface 


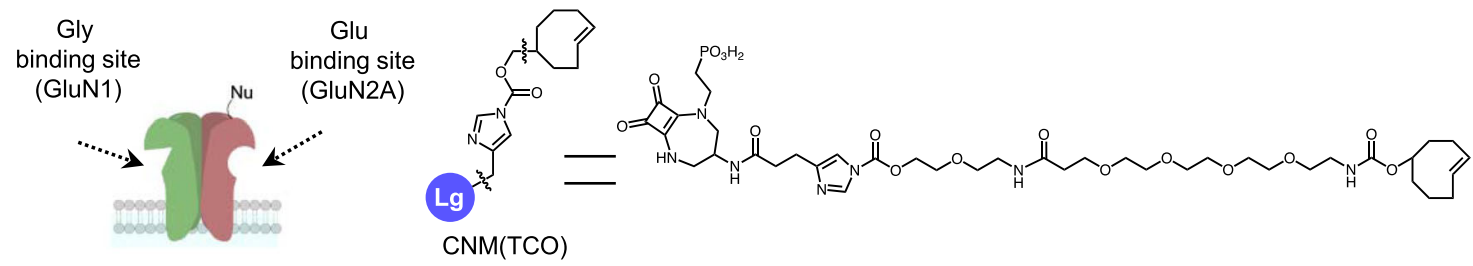

b
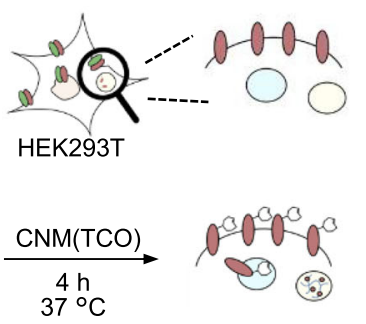

c

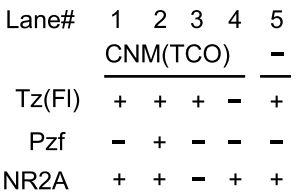

(kDa) 250

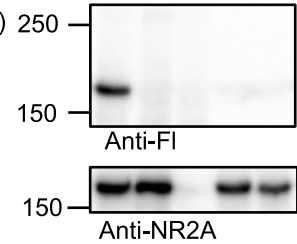

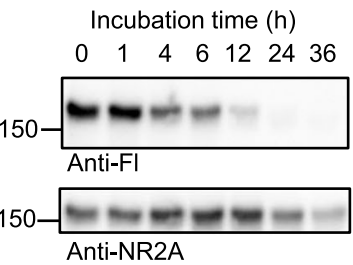

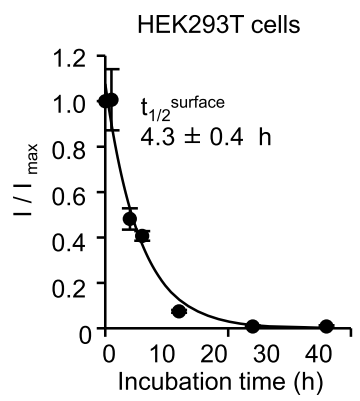

d

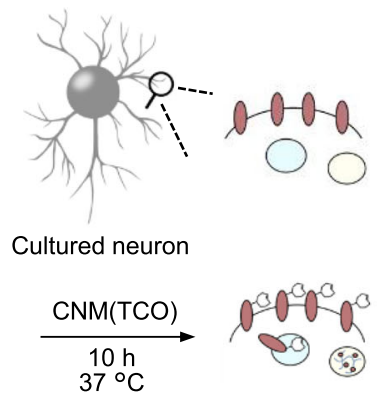

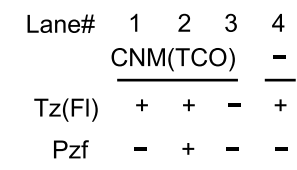

(kDa)

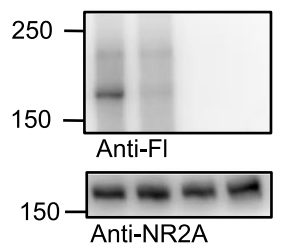

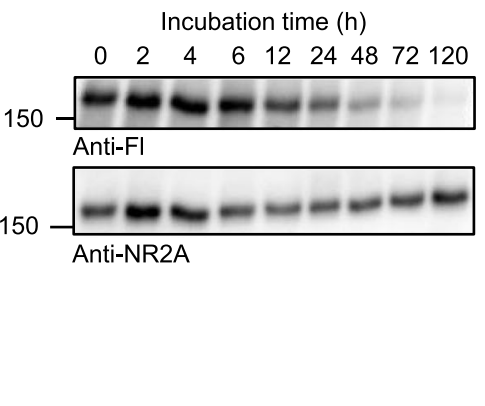

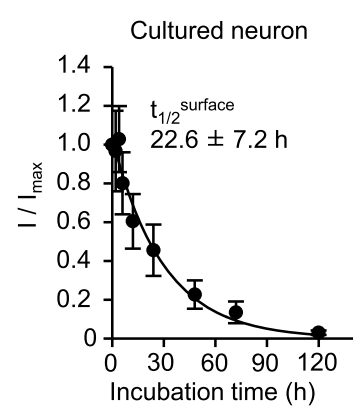

Fig. 6 The two-step labeling of cell-surface NMDARs in HEK293T cells and neurons. a Chemical structure of CNM(TCO) for two-step labeling reagent for NMDARs. Gly glycine. b Two-step labeling of cell-surface NMDARs ectopically expressed in HEK293T cells. In left, schematic illustration is shown. In right, western blotting analyses after the two-step labeling are shown. HEK293T cells transfected with NR1-1 and NR2A or vector control were treated with $10 \mu \mathrm{M}$ CNM(TCO) for $4 \mathrm{~h}$ followed by the addition of $1 \mu \mathrm{M} \mathrm{Tz}(\mathrm{FI})$ for $5 \mathrm{~min}$ in the presence or absence of $250 \mu \mathrm{M}$ Pzf in culture medium at $37^{\circ} \mathrm{C}$. The cell lysates were analyzed by western blotting using anti-fluorescein or anti-NR2A antibody. c Determination of $t_{1 / 2}$ surface of NMDARs in HEK293T cells by western blotting. In left, representative results of western blotting are shown. In right, time-course of the labeled band is shown ( $n=3$ biological replicates). d Two-step labeling of cell-surface NMDARs endogenously expressed in cultured cortical neurons. In left, schematic illustration is shown. In right, western blotting analyses after the two-step labeling are shown. The cultured cortical neurons were treated with $10 \mu \mathrm{M}$ CNM(TCO) for $10 \mathrm{~h}$ followed by the addition of $1 \mu \mathrm{M} \mathrm{Tz}(\mathrm{FI})$ for $5 \mathrm{~min}$ in the presence or absence of $250 \mu \mathrm{M}$ Pzf in culture medium at $37^{\circ} \mathrm{C}$. The cell lysates were analyzed by western blotting using antifluorescein or anti-NR2A antibody. e Determination of $t_{1 / 2}$ surface of NMDARs in neurons by western blotting. In left, representative results of western blotting are shown. In right, time-course of the labeled band is shown ( $n=3$ biological replicates). Data are represented as mean \pm s.e.m.

AMPARs that are endogenously expressed in neurons. In addition, the endogenous AMPARs were successfully visualized using probes such as Alexa 647 or SeTau-647, using Tz(Ax647) or $\mathrm{Tz}$ (ST647), respectively (Supplementary Fig. 14b, c).

Next, we quantified the surface distribution of AMPARs in neurons using fluorescence lifetime imaging microscopy (FLIM). In this method, the fluorescence decay curve in each pixel is analyzed by fitting it to a multi-exponential function, and the target fluorescence lifetime $(\tau)$ component can be extracted to quantify the fluorescence of interest without any background. Here, we used $\mathrm{Tz}$ (ST647) to visualize surface AMPARs because SeTau-647 has a unique fluorescence lifetime and high photostability 39,40 . The typical FLIM image for a lifetime fraction ( $\tau=2.4 \pm 0.1 \mathrm{~ns}$ ) corresponding to SeTau-647 is shown in Fig. 4 d, which revealed that surface AMPARs in spines are 3.3 times more concentrated than those in dendrites.
Trafficking analyses of endogenous AMPARs in neurons. AMPARs are dynamically regulated at synapses, which underlie activity-dependent neuronal plasticity. Molecular biology or biochemical methods, such as the genetic incorporation of fluorescent proteins, surface biotinylation assays, and metabolic incorporation of radioisotopes, have revealed the diffusion dynamics $^{42}$, recycling process 5 , and half-life ${ }^{43,44}$ of AMPARs, respectively. Although powerful, these methods are highly specialized for analyzing each process (see the Discussion for detail). However, we now have a rapid method to selectively label cellsurface AMPARs in neurons under physiological temperature in culture medium. We therefore applied the two-step labeling method to analyze AMPAR trafficking over a long period.

Prior to analyzing the AMPAR trafficking, we examined the influence of the two-step labeling process on the viability of primary cultured neurons by comparing it with our original 
CAM2 labeling. In immature neurons (day in vitro [DIV] 4), neither the original CAM2 labeling nor the two-step labeling affected neuronal viability after $24 \mathrm{~h}$ of labeling (Supplementary Fig. 15). However, in mature neurons (DIV 12), where iGluRs are highly expressed, viability was decreased after $3 \mathrm{~h}$ of labeling with the original CAM2 labeling, and neurons were severely damaged after $24 \mathrm{~h}$ of the original labeling. The main reason for the lowered viability may be ascribed to the experimental procedure rather than the CAM2 reagents, because similar neuronal damage was observed when the growth medium was exchanged for serum-free medium. In contrast, in the case of the two-step labeling method, where CAM2(TCO) was directly added into the growth medium at $37^{\circ} \mathrm{C}$, negligible damage was observed even after $24 \mathrm{~h}$ of the two-step labeling, indicating that this method is suitable for analyzing AMPARs over long periods in neurons. Besides, we examined the effects of CAM2(TCO) labeling on neuronal function. Neither the surface amount nor the synaptic fraction of AMPARs and NMDARs were affected by CAM2(TCO) labeling for $10 \mathrm{~h}$ (Supplementary Fig. 17a, b). Association between GluA2 and the accessary protein, TARP $\gamma 8$ (ref. ${ }^{45}$ ) was also unaffected by CAM2(TCO) labeling (Supplementary Fig. 17c). Although the homeostatic phosphorylation of ERK and CREB was not significantly but slightly decreased, phosphorylation levels of AMPAR (GluA1) and NMDAR (GluN1) were not influenced by the CAM2(TCO) labeling (Supplementary Fig. 17d).

We then analyzed the trafficking of endogenous AMPARs in neurons over a long period using the two-step labeling method. As described in the previous section, confocal microscopic imaging was able to clearly visualize cell-surface AMPARs in neurons in the two-step labeling method (Fig. 4c). However, synapses are very narrow (200-500 $\mathrm{nm}$ diameter), and it is thus difficult to analyze AMPAR trafficking in detail using optical microscopy. We therefore applied biochemical methods for analyzing the $t_{1 / 2}$ surface of AMPARs, including remaining and recycled components (Fig. 5a). As shown in Fig. 5b, the $t_{1 / 2}$ surface was calculated to be $33.2 \pm 5.1 \mathrm{~h}$, which was markedly longer than the $t_{1 / 2}$ surface in HEK293T cells $(5.3 \pm 0.3 \mathrm{~h})$.

To explore the difference in the $t_{1 / 2}$ surface values, we focused on the trafficking of internalized AMPARs. We analyzed the intracellular and surface percentages of TCO-AMPARs after $10 \mathrm{~h}$ of incubation with CAM2(TCO). The intracellular percentage $(10.3 \pm 1.7 \%)$ was markedly smaller than the surface percentage $(90.4 \pm 0.6 \%)$, and these values were largely similar to those obtained after $10 \mathrm{~h}$ of labeling in HEK293T cells (Fig. 5c and Supplementary Fig. 18). We next evaluated the recycling process of internalized AMPARs, as follows. The cultured neurons were labeled with CAM2(TCO) for $10 \mathrm{~h}$ in the culture medium at $37^{\circ} \mathrm{C}$, and $\mathrm{Tz}(\mathrm{Fl})$ was then added to the culture medium for $5 \mathrm{~min}$ as a first pulse to mask the surface TCOAMPARs (Fig. 5d). The neurons were further incubated for 15 min in the culture medium at $37^{\circ} \mathrm{C}$, and then $\mathrm{Tz}(\mathrm{Ax} 647)$ was added to the culture medium as a second pulse to label the recycled TCO-AMPARs. As shown in Fig. 5e, western blotting using anti-Alexa 647 antibodies clearly detected recycled AMPARs. The percentage was determined to be $12.5 \pm 2.8 \%$ compared with the surface AMPARs, suggesting that most of internalized AMPARs were recycled during this short period. In contrast, the recycled fraction was almost undetectable in HEK293T cells (Supplementary Fig. 20). These data indicate that AMPARs are constantly recycled via endocytosis and exocytosis in neurons, which may be the molecular basis for the long lifetimes of AMPARs (Fig. 5f, g).

Two-step labeling and trafficking analyses of NMDARs. The NMDARs are another essential family of iGluRs, and form heterotetramers composed of GluN1, GluN2A-D, and GluN3 in neurons. Pharmacological features of NMDARs are different from those of AMPARs. GluN2A-D recognize glutamate, whereas GluN1 and GluN3 recognize glycine or D-serine, as endogenous ligands ${ }^{4}$. Here, we designed a ligand-directed twostep labeling reagent targeted for GluN2A using the selective antagonist perzinfotel (Pzf) as the ligand moiety (Fig. 6a) ${ }^{46}$. This reagent was termed "chemical NMDAR modification (TCO)" (CNM(TCO)).

To confirm the selective labeling of GluN2A, the two-step labeling method was first examined in HEK293T cells transiently co-expressed with GluN1 and GluN2A. Western blotting analyses of cell lysate showed a prominent band around $180 \mathrm{kDa}$, corresponding to the molecular weight of GluN2A (see lane 1 in Fig. 6 b). The $180 \mathrm{kDa}$ band was not detected under the control conditions (see lanes $2-5$ in Fig. 6b and Supplementary Fig. 21), suggesting that this band corresponds to labeled GluN2A. The $t_{1 / 2}$ surface of cell-surface NMDARs was quantitatively analyzed using western blotting by focusing on the labeled GluN2A band. As shown in Fig. $6 \mathrm{c}$, the $t_{1 / 2}$ surface was calculated to be $4.3 \pm 0.4 \mathrm{~h}$, which was slightly shorter than that of AMPARs in HEK293T cells.

Next, we examined the chemical labeling of endogenous NMDARs in cultured neurons using CNM(TCO). As shown in Fig. 6d, western blotting showed a prominent band around $180 \mathrm{kDa}$, which was negligible in the co-presence of Pzf, indicating the successful labeling of endogenous NMDARs. Similar to AMPAR labeling, the time-course of NMDAR labeling clearly indicated that chemical labeling occurred more efficiently at $37^{\circ} \mathrm{C}$ than at $17^{\circ} \mathrm{C}$ (Supplementary Fig. 22a). However, the concentration dependency of CNM(TCO) for NMDAR labeling was different from that of CAM2(TCO) for AMPARs. As shown in Supplementary Fig. 22b, the labeled bands were not saturated in the $0-10 \mu \mathrm{M}$ range, indicating that the affinity of $\mathrm{CNM}(\mathrm{TCO})$ was lower than that of CAM2(TCO). Importantly, CNM(TCO) labeled NMDAR with minimal disturbance to neuronal functions, including the constitutive phosphorylation of ERK and CREB (Supplementary Fig. 23). We next analyzed NMDAR trafficking using two-step labeling via biochemical methods in neurons. As shown in Fig. 6e, the $t_{1 / 2}$ surface of endogenous NMDARs in cultured neurons was $22.6 \pm 7.2 \mathrm{~h}$, which was substantially longer than in HEK293T cells. However, the value was shorter than that of endogenous AMPARs in neurons $(33.2 \pm 5.1 \mathrm{~h})$. Thus, two-step labeling was successfully applied to NMDARs, another important family of iGluRs, by changing the selective ligands. In addition, we revealed that the lifetime of surface NMDARs is shorter than that of AMPARs in neurons.

\section{Discussion}

We described a bioorthogonal two-step labeling method for the selective modification of chemical probes to cell-surface AMPARs. This method can be used under neuronal culture conditions. In our previous method, fluorescein was directly labeled to cell-surface AMPARs using CAM2(Fl) in serum-free medium at $17^{\circ} \mathrm{C}$ (ref. ${ }^{26}$ ). Although the dynamics of cell-surface AMPARs have been successfully tracked over a short period after labeling, the present study revealed that this labeling condition affects the viability of mature neurons after $24 \mathrm{~h}$ of labeling. In contrast, the present bioorthogonal two-step labeling method negligibly affected the cell viability of neurons. Our method therefore allows the quantitative analysis of AMPAR trafficking for over $120 \mathrm{~h}$ after labeling. The present investigation also revealed that the homeostatic phosphorylation of ERK and CREB was slightly decreased by CAM2(TCO) but not CNM(TCO) treatment. Considering the high affinity of CAM2(TCO) for AMPAR, this influence may be reduced when neurons are treated with low concentration of CAM2(TCO). 
Many studies have focused on analyzing AMPAR trafficking in neurons using biochemical methods. To study trafficking over short periods (less than $30 \mathrm{~min}$ ), surface biotinylation assays, where cleavable (disulfide-linked) biotin is randomly labeled on the cell surface, have conventionally been used. To date, both endocytosis and exocytosis of AMPARs have been investigated in cultured neurons using this method. However, this method is not suitable for analyzing AMPAR trafficking over a long period, because the biotinylation reaction must be conducted in serumfree medium at $4{ }^{\circ} \mathrm{C}$; this affects neuronal viability, as we demonstrated in the present study. The long-term process of AMPAR trafficking, including decomposition, has been previously analyzed using the metabotropic incorporation of a radioisotope $\left.{ }^{35} \mathrm{~S}\right]$-labeled amino acid, or SILAC (stable isotope labeling with amino acids in cell culture), for mass spectrometry analyses ${ }^{43,44,47}$. Although isotope labeling can be conducted under cell culture conditions, these methods are not suitable for analyzing AMPAR trafficking over short periods. In contrast, our bioorthogonal two-step labeling selectively and rapidly modifies target iGluRs under cell culture conditions, and this allows us to analyze receptor trafficking over both short and long periods. Moreover, both biotinylation assays and metabotropic isotope labeling methods require the solubilization of iGluRs by mild detergents for pull-down or immunoprecipitation assays, because cellular proteins are randomly labeled in these methods. This step is problematic for the quantitative analysis of iGluRs, because iGluRs such as NMDARs are mainly localized in the postsynaptic density (PSD), where it is difficult for proteins to be solubilized by mild detergents. This peculiar feature hampers the quantitative analysis of iGluR trafficking by conventional biochemical methods. In contrast, purification steps are not required after labeling in our two-step labeling method. That is, in our method, all labeled proteins in the neurons can be analyzed quantitatively after denaturing in Laemmli sample buffer. This allows for the quantitative analysis of iGluRs contained in the PSD fraction.

The selective visualization of cell-surface iGluRs is essential for analyzing their trafficking and distribution. One potential method involves the use of antibodies that selectively recognize the extracellular regions of iGluRs. However, these antibodies are very limited, and in most cases their selectivity is insufficient. Positron emission tomography imaging would be another candidate for visualizing native AMPARs ${ }^{4}$. However, this method would not be useful for trafficking studies due to its low resolution (1-2 mm). Here, we demonstrated that various kinds of chemical probes could be used to selectively and rapidly label endogenous cellsurface iGluRs in neurons using bioorthogonal two-step labeling. The selective labeling of SeTau-647, a middle-sized molecule with a long fluorescence lifetime and high photostability, allowed the quantitative analyses of cell-surface AMPAR distribution in neurons using FLIM. These analyses revealed a three-fold concentration of cell-surface AMPARs in spines compared with dendrites. As a future direction, cell-surface SeTau-647 labeling would be utilized for single molecule tracking ${ }^{40}$ or super resolution imaging of synaptic AMPARs. Moreover, the second step IEDDA reaction can be applied to label polymers or nanoparticles, to allow the distribution of iGluRs to be analyzed in more detail using electron microscopy in the future.

Previous studies using radiolabeling methods indicated that the half-lives of synaptic proteins are 1-2 days ${ }^{43,44}$. Consistent with previous results, our present investigation demonstrated that the halflives of AMPARs and NMDARs are 33.2 and $22.6 \mathrm{~h}$, respectively, in cultured neurons. However, these values were significantly longer than those obtained in HEK293T cells. A plausible explanation for the differences between neurons and HEK293T cells may involve the formation of the macromolecular protein complexes of iGluRs. In the case of AMPARs, many binding partners such as transmembrane
AMPAR regulatory proteins, synapse-associated protein $97 \mathrm{kDa}$, and glutamate receptor-interacting protein, which are selectively expressed in neurons, control the recycling of AMPARs and/or their stabilization at synapses ${ }^{45,49,50}$. Differences in phosphorylation levels may be another possible explanation. Activity-dependent phosphorylation by CaMKII or PKA contributes to the recycling and cellsurface insertion of AMPARs ${ }^{51,52}$. In addition, ubiquitination or deubiquitination via Nedd4 or USP46, respectively, may be considered $^{53,54}$. In most cases, the contributions of these accessory proteins and post-translational modifications to AMPAR trafficking have been evaluated using genetic approaches, such as overexpression of an AMPAR subunit tagged with $\mathrm{pH}$-sensitive SEP on the $\mathrm{N}$ terminus. However, in some cases, complementary genetic experiments using knock-in or knock-out mice of the target gene have not supported the data ${ }^{19}$. Considering the heterotetrameric formation of AMPARs by the GluA1-4 subunits, and considering that each subunit has inherent roles, conflicting findings may be ascribed to the formation of non-native tetramers by the overexpression of single AMPAR subunits in neurons. In contrast, the present two-step labeling method can be used to visualize native iGluRs under physiological temperature in culture medium. Thus, this method can contribute to our understanding of the physiological and pathophysiological roles of iGluR trafficking in neurons.

\section{Methods}

Synthesis. All synthesis procedures and compound characterizations are described in Supplementary Methods.

General methods for biochemical and biological experiments. SDS-PAGE and western blotting were carried out using a BIO-RAD Mini-Protean III electrophoresis apparatus. Samples were applied to SDS-PAGE and electrotransferred onto polyvinylidene fluoride membranes (BIO-RAD), followed by blocking with $5 \%$ nonfat dry milk in Tris-buffered saline containing $0.05 \%$ Tween 20 . Primary antibody was indicated in each experimental procedure, and anti-rabbit IgG-HRP conjugate (CST, 7074S, 1:3,000) or anti-mouse IgG-HRP conjugate (CST, 7076S, $1: 3,000)$ was utilized as the secondary antibody. Chemiluminescent signals generated with ECL Prime (GE Healthcare) were detected with a Fusion Solo S imaging system (Vilber Lourmat).

Animals. Pregnant ICR mice and pregnant Sprague Dawley rats maintained under specific pathogen-free conditions were purchased from Japan SLC, Inc (Shizuoka, Japan). The animals were housed in a controlled environment $\left(23 \pm 1{ }^{\circ} \mathrm{C}, 12 \mathrm{~h}\right.$ light/dark cycle) and had free access to food and water, according to the regulations of the Guidance for Proper Conduct of Animal Experiments by the Ministry of Education, Culture, Sports, Science, and Technology of Japan. All experimental procedures were performed in accordance with the National Institute of Health Guide for the Care and Use of Laboratory Animals, and were approved by the Institutional Animal Use Committees of Kyoto University or Nagoya University.

Expression of AMPARs or NMDARs in HEK293T cells. HEK293T cells (ATCC) were cultured in Dulbecco's modified Eagle's medium (DMEM)-GlutaMAX (Invitrogen) supplemented with $10 \%$ dialyzed FBS (Invitrogen), penicillin (100 units $\left.\mathrm{ml}^{-1}\right)$, and streptomycin $\left(100 \mu \mathrm{g} \mathrm{ml}^{-1}\right)$, and incubated in a $5 \% \mathrm{CO}_{2}$ humidified chamber at $37^{\circ} \mathrm{C}$. Cells were transfected with a plasmid encoding rat GluA2 $\left(\mathrm{GluA} 2^{\text {flip }}(\mathrm{Q})\right)^{26}$ or the control vector pCAGGS (kindly provided by Dr. H. Niwa from RIKEN) using Lipofectamine 2000 (Invitrogen) according to the manufacturer's instructions, and subjected to labeling experiments after $36-48 \mathrm{~h}$ of the transfection. For NMDAR expression, cells were transfected with plasmids encoding rat GluN1-1 ${ }^{55}$ and rat GluN2 $\mathrm{A}^{55}$, and $30 \mu \mathrm{M}$ MK-801 (Funakoshi) was added to the culture medium to suppress cell death.

Two-step labeling of AMPARs or NMDARs in HEK293T cells. For the first step labeling of AMPARs, HEK293T cells transfected with GluA2 were treated with $2 \mu \mathrm{M}$ CAM2(TCO) in the absence or presence of $50 \mu \mathrm{M}$ NBQX in the culture medium at $37^{\circ} \mathrm{C}$ for $4 \mathrm{~h}$. For the second step labeling, the culture medium was removed, and $1 \mu \mathrm{M} \mathrm{Tz}(\mathrm{Fl})$ in $\mathrm{PBS}$ was added for $5 \mathrm{~min}$ at room temperature. To quench excess $\mathrm{Tz}(\mathrm{Fl}), 1 \mu \mathrm{M}$ TCO-PEG4-COOH in PBS was added.

For western blot analyses of labeled AMPARs, labeled cells were washed three times with PBS, lysed with radio immunoprecipitation assay (RIPA) buffer containing $1 \%$ protease inhibitor cocktail (Nacalai tesque), and mixed with $5 \times$ Laemmli sample buffer containing $250 \mathrm{mM}$ DTT. Western blotting analyses were performed as described in "General methods for biochemical and biological experiments." The Fl-labeled GluA2 was detected using rabbit anti-fluorescein 
antibody (abcam, ab19491, 1:3,000). GluA2 was detected using a rabbit antiGluA2/3 antibody (Millipore, 07-598, 1:3,000).

In the case of two-step labeling of NMDARs, HEK293T cells transfected with GluN1-1 and GluN2A were treated with $10 \mu \mathrm{M} C N M(T C O)$ in the absence or presence of $250 \mu \mathrm{M} \mathrm{Pzf}$ in the culture medium at $37^{\circ} \mathrm{C}$ for $4 \mathrm{~h}$. The second step labeling and subsequent western blotting were performed as described above. Immunodetection of GluN2A was performed with a rabbit anti-NR2A antibody (Millipore, 07-632, 1:1,000)

CAM2(TCO), CNM(TCO) and Tz-probes were stored in DMSO solution. The stock solutions were kept in deep freezer $\left(-80^{\circ} \mathrm{C}\right)$ to prevent decomposition.

Enzymatic deglycosylation of AMPARs expressed in HEK293T cells. GluA2 expressing HEK293T cells were labeled as described in "Two-step labeling of AMPARs or NMDARs in HEK293T cells." The labeled cells were washed three times with PBS and lysed in PBS containing 1\% triton X-100, 0.6\% SDS, and 1\% protease inhibitor cocktail for $30 \mathrm{~min}$ at $37^{\circ} \mathrm{C}$. The lysates were diluted (2.0-fold) in sodium phosphate buffer $(50 \mathrm{mM}, \mathrm{pH} 7.5)$ containing $2 \%$ NP40 and $100 \mathrm{mM}$ DTT. PNGase F (New England Biolabs) were used at 1,000 units/100 $\mu$ l of lysate and incubated overnight at $37^{\circ} \mathrm{C}$. The samples were subjected to western blotting analyses as described in "Two-step labeling of AMPARs or NMDARs in HEK293T cells." In this experiment, after western blotting using anti-Fl antibody, the membrane was stripped with stripping buffer $(250 \mathrm{mM}$ glycine $(\mathrm{pH}=2.5)$ and $1 \%$ SDS) and reprobed with the anti-GluA2/3 antibody.

\section{Confocal live cell imaging of labeled AMPARs in HEK293T cells}

HEK293T cells were co-transfected with $\mathrm{GluA}^{\text {flip }}(\mathrm{Q})$ and mCherry- $\mathrm{F}^{26}$ as a transfection marker. First step labeling was performed as describe in "Two-step labeling of AMPARs or NMDARs in HEK293T cells." For the second step labeling, after removal of the culture medium, $100 \mathrm{nM} \mathrm{Tz}(\mathrm{Ax} 488)$ was treated for $5 \mathrm{~min}$ in HBS $(20 \mathrm{mM}$ HEPES, $107 \mathrm{mM} \mathrm{NaCl}, 6 \mathrm{mM} \mathrm{KCl}, 2 \mathrm{mM} \mathrm{CaCl}_{2}$, and $1.2 \mathrm{mM} \mathrm{MgSO}_{4}$ at $\mathrm{pH} 7.4$ ) at room temperature and washed three times with HBS. Confocal live imaging was performed with a confocal microscope (LSM900, Carl Zeiss) equipped with a $63 \times$, numerical aperture $(\mathrm{NA})=1.4$ oil-immersion objective. Fluorescence images were acquired by excitation at $405,488,561$, or $640 \mathrm{~nm}$ derived from diode lasers.

For studying the reaction kinetics of tetrazine ligation to cell surface AMPARs, after first step labeling, cells were then incubated with $300 \mathrm{nM} \mathrm{Tz}(\mathrm{Ax} 488)$ at room temperature and imaged at specified time points by confocal microscopy. To quantify the fluorescence intensity of the membrane at each time point, mCherry-F positive cells $(n=6)$ were selected and the average signal intensity of ROIs set on the membrane was calculated by ZEN blue software (Carl Zeiss). After subtracting background fluorescence, the averaged membrane intensity was defined as $F$ at each time point $\left(F_{\text {MAX }}\right.$ was defined as $F$ at $20 \mathrm{~min}$ ). The membrane intensity was fitted with KaleidaGraph (Synergy software) using following equation (1): $F=a+b\left(1-e^{-\mathrm{ct}}\right)$.

For trafficking analyses, after first step labeling, $100 \mathrm{nM} \mathrm{Tz}(\mathrm{Ax} 488)$ was added in DMEM-GlutaMAX for $5 \mathrm{~min}$. The cells were washed three times in DMEMGlutaMAX and incubated for $0,1,4$, and $8 \mathrm{~h}$ in growth medium at $37^{\circ} \mathrm{C}$. Live cell imaging was performed with a confocal microscope. To quantify the fluorescence intensity of the membrane at each time point, mCherry-F positive cells were selected and the average signal intensity of ROIs set on the membrane was calculated by ZEN blue software after subtracting background fluorescence. The averaged membrane intensity was defined as $F$ at each time point. The membrane intensity was fitted with KaleidaGraph using following equation (2): $F=a+b \cdot e^{-c t}$, and the offset value $(a)$ was set equal to zero. The $t_{1 / 2}$ was defined as $t_{1 / 2}=\ln (2) / c$.

For co-staining with LysoTracker Red DND-99 (Invitrogen), after first step labeling, the cells were treated with $50 \mathrm{nM}$ LysoTracker Red DND-99 for $30 \mathrm{~min}$ at $37^{\circ} \mathrm{C}$ in culture medium, and then treated with $100 \mathrm{nM} \mathrm{Tz}(\mathrm{Ax} 488)$ in culture medium for $5 \mathrm{~min}$. After washing three times with culture medium or subsequent incubation for $8 \mathrm{~h}$, cells were imaged using a confocal microscope.

Half-life studies of AMPARs by western blotting in HEK293T cells. Schematic illustration of the experiments is shown in Fig. 3 d. For determining $t_{1 / 2}$ surface (path1 in Fig. 3d), first step labeling was conducted as described in "Two-step labeling of AMPARs or NMDARs in HEK293T cells." After medium exchange for removal of the labeling reagents, the cells were incubated for $0,1,4,6,12,24$, and $36 \mathrm{~h}$. The cells were treated with $1 \mu \mathrm{M} \mathrm{Tz}(\mathrm{Fl})$ in $\mathrm{PBS}$ for $5 \mathrm{~min}$, and excess $\mathrm{Tz}(\mathrm{Fl})$ was quenched by addition of $1 \mu \mathrm{M}$ TCO-PEG4-COOH in PBS.

For determining $t_{1 / 2}$ life (path-2 in Fig. $3 \mathrm{~d}$ ), after first step labeling, cells were washed three times with culture medium and treated with $1 \mu \mathrm{M} \mathrm{Tz}(\mathrm{Fl})$ in culture medium for $5 \mathrm{~min}$. Excess $\mathrm{Tz}(\mathrm{Fl})$ was quenched by addition of $1 \mu \mathrm{M}$ TCO-PEG4$\mathrm{COOH}$ in culture medium. Cells were then incubated for $0,1,4,6,12,24$, and $36 \mathrm{~h}$ and washed three times with PBS. Cell lysis and western blotting were performed as described in "Two-step labeling of AMPARs or NMDARs in HEK293T cells."

The target bands were manually selected, and the intensity were calculated with Fusion software (Vilber Lourmat), background intensity was manually subtracted by cutting the minimal intensity in the selected area. The half-life was calculated by curve fitting using KaleidaGraph and following equation (3): $I=a+b \cdot e^{-c t}$, and the offset value (a) was set equal to zero. The $t_{1 / 2}$ was defined as $t_{1 / 2}=\ln (2) / c$.
Intracellular and surface ratio of labeled AMPARs in HEK293T cells. Schematic illustration of the experiments is shown in Fig. 3g. For determining labeled AMPARs on cell surface (path-1 in Fig. 3g), after first step labeling as describe in "Two-step labeling of AMPARs or NMDARs in HEK293T cells," the cells were treated with $1 \mu \mathrm{M} \mathrm{Tz}(\mathrm{Fl})$ for $5 \mathrm{~min}$ in PBS at room temperature. To quench excess $\mathrm{Tz}(\mathrm{Fl}), 1 \mu \mathrm{M}$ TCO-PEG4-COOH in PBS was added and lysed with RIPA buffer containing $1 \%$ protease inhibitor cocktail for $30 \mathrm{~min}$ at $4{ }^{\circ} \mathrm{C}$.

For determining intracellular labeled AMPARs (path-2 in Fig. 3g), after first step labeling, $1 \mu \mathrm{M} \mathrm{Tz}(\mathrm{Ax} 647)$ was treated for $5 \mathrm{~min}$ for masking of cell-surface TCO-labeled AMPARs. After cell lysis using RIPA buffer, the lysate was reacted with $0.3 \mu \mathrm{M} \mathrm{Tz}(\mathrm{Fl})$ for $30 \mathrm{~min}$ at room temperature. Excess $\mathrm{Tz}(\mathrm{Fl})$ was quenched by addition of $1 \mu \mathrm{M}$ TCO-PEG4-COOH in the cell lysate.

For preparing whole-cell-labeling fraction (path-3 in Fig. 3g), after first step labeling, the cells were lysed with RIPA buffer containing $1 \%$ protease inhibitor cocktail for $30 \mathrm{~min}$ at $4{ }^{\circ} \mathrm{C}$. The lysate was reacted with $0.3 \mu \mathrm{M} \mathrm{Tz}(\mathrm{Fl})$ for $30 \mathrm{~min}$ at room temperature. Excess $\mathrm{Tz}(\mathrm{Fl})$ was quenched by addition of $1 \mu \mathrm{M}$ TCO-PEG4$\mathrm{COOH}$ in the cell lysate.

Western blotting was performed as described in "Half-life studies of AMPARs by western blotting in HEK293T cells." The target bands were manually selected, and the intensity were calculated with ImageJ software, background intensity was manually subtracted by selecting a region with no bands from the same lane. In more detail, the band intensity was determined as described below:

$($ target intensity $)-($ target area $) /($ background area $) \times($ background intensity $)$

Preparation of primary cortical neuronal culture. Twenty-four-well plates (BD Falcon) were coated with poly-D-lysine (Sigma-Aldrich), and washed with sterile $\mathrm{dH}_{2} \mathrm{O}$ three times. Cerebral cortices from 16-day-old ICR mouse embryos were aseptically dissected and digested with $0.25 \mathrm{w} / \mathrm{v} \%$ trypsin (Nacalai tesque) for $20 \mathrm{~min}$ at $37^{\circ} \mathrm{C}$. The cells were re-suspended in Neurobasal Plus medium supplemented with $10 \%$ FBS, penicillin (100 units $/ \mathrm{ml})$, and streptomycin $(100 \mu \mathrm{g} / \mathrm{ml})$ and filtered by Cell Strainer $(100 \mu \mathrm{m}$, Falcon) and centrifuged at $1,000 \mathrm{rpm}$ for $5 \mathrm{~min}$. The cells were resuspended in Neurobasal Plus medium supplemented with $2 \%$ of B-27 Plus Supplement, $1.25 \mathrm{mM}$ GlutaMAX I (Invitrogen), penicillin (100 units $/ \mathrm{ml}$ ), and streptomycin $(100 \mu \mathrm{g} / \mathrm{ml})$ and plated at a density of $2 \times 10^{5}$ cells on the 24 -well plate. The cultures were maintained at $37^{\circ} \mathrm{C}$ in a $95 \%$ air and $5 \% \mathrm{CO}_{2}$ humidified incubator. Culture medium was replaced every 3 or 4 days and the neurons were used at 12-15 DIV.

Two-step labeling of AMPARs or NMDARs in cultured neurons. To label endogenous AMPARs, $12 \mu \mathrm{M}$ CAM2(TCO) in $100 \mu \mathrm{l}$ of the culture medium with or without $300 \mu \mathrm{M}$ NBQX was gently added to the cortical neurons cultured in 500 $\mu \mathrm{l}$ medium on 24-well plates to a final concentration of $2 \mu \mathrm{M}$ CAM2(TCO) and 50 $\mu \mathrm{M}$ NBQX. The cells were incubated for $10 \mathrm{~h}$ at $37^{\circ} \mathrm{C}$. For the second step, the culture medium was removed and the cells were treated with $1 \mu \mathrm{M} \mathrm{Tz}(\mathrm{Fl})$ for $5 \mathrm{~min}$ in PBS at room temperature. To quench excess $\mathrm{Tz}(\mathrm{Fl}), 1 \mu \mathrm{M}$ TCO-PEG4-COOH in PBS was added. Western blot analyses of labeled AMPARs were performed as described in "Two-step labeling of AMPARs or NMDARs in HEK293T cells."

To label endogenous NMDARs, $60 \mu \mathrm{M}$ CNM(TCO) in $100 \mu \mathrm{l}$ of growth medium with or without $1.5 \mathrm{mM}$ Pzf was gently added to the cortical neurons cultured in $500 \mu \mathrm{l}$ medium on 24-well plates to a final concentration of $10 \mu \mathrm{M}$ $\mathrm{CNM}(\mathrm{TCO})$ and $50 \mu \mathrm{M}$ Pzf. The cells were incubated for $10 \mathrm{~h}$ at $37^{\circ} \mathrm{C}$. The second step labeling and subsequent western blotting was performed as described above.

Half-life studies of endogenous AMPARs or NMDARs in cultured neurons Schematic illustration of the experiments is shown in Fig. 5a. For determining $t_{1 / 2}$ surface, after first step labeling as describe in "Two-step labeling of AMPARs or NMDARs in cultured neurons," the cells were incubated for $0,2,4,6,12,24,48,72$, and $120 \mathrm{~h}$. For the second step, the culture medium was removed and the cells were treated with $1 \mu \mathrm{M} \mathrm{Tz}(\mathrm{Fl})$ for $5 \mathrm{~min}$ in PBS at room temperature. To quench excess $\mathrm{Tz}$ (Fl), $1 \mu \mathrm{M}$ TCO-PEG4-COOH in PBS was added and washed three times with PBS Cell lysis and western blotting were performed as described in "Two-step labeling of AMPARs or NMDARs in HEK293T cells." The immunodetection of GluA2 was conducted with a rabbit anti-GluA2 antibody (abcam, ab20673, 1:3,000). Quantification of the band intensity and calculation of the half-time was calculated described as in "Half-life studies of AMPARs by western blotting in HEK293T cells."

Intracellular and surface ratio of labeled AMPARs in cultured neurons. Schematic illustration of the experiments is shown in Fig. 3g. For determining labeled AMPARs on cell surface (path-1 in Fig. 3g), after first step labeling as describe in "Two-step labeling of AMPARs or NMDARs in cultured neurons," the cells were treated with $1 \mu \mathrm{M} \mathrm{Tz}(\mathrm{Fl})$ for $5 \mathrm{~min}$ in PBS at room temperature. To quench excess $\mathrm{Tz}(\mathrm{Fl}), 1 \mu \mathrm{M}$ TCO-PEG4-COOH in PBS was added and lysed with RIPA buffer containing $1 \%$ protease inhibitor cocktail for $30 \mathrm{~min}$ at $4{ }^{\circ} \mathrm{C}$.

For determining intracellular labeled AMPARs (path-2 in Fig. 3g), after first step labeling, $1 \mu \mathrm{M} \mathrm{Tz}(\mathrm{Ax} 647)$ was treated for $5 \mathrm{~min}$ for masking of cell-surface TCO-labeled AMPARs. After cell lysis using RIPA buffer containing $1 \%$ protease inhibitor cocktail for $30 \mathrm{~min}$ at $4{ }^{\circ} \mathrm{C}$, the lysate was reacted with $0.3 \mu \mathrm{M} \mathrm{Tz}(\mathrm{Fl})$ for $30 \mathrm{~min}$ at room temperature. Excess $\mathrm{Tz}(\mathrm{Fl})$ was quenched by addition of $1 \mu \mathrm{M}$ TCO-PEG4-COOH in the cell lysate. 
For preparing whole-cell-labeling fraction (path-3 in Fig. 3g), after first step labeling, the cells were lysed with RIPA buffer containing $1 \%$ protease inhibitor cocktail for $30 \mathrm{~min}$ at $4{ }^{\circ} \mathrm{C}$. The lysate was reacted with $0.3 \mu \mathrm{M} \mathrm{Tz}(\mathrm{Fl})$ for $30 \mathrm{~min}$ at room temperature. Western blotting was performed as described in "Two-step labeling of AMPARs or NMDARs in HEK293T cells." Quantification of the band intensity and calculation of the ratio was conducted as described in "Intracellular and surface ratio of labeled AMPARs in HEK293T cells."

Quantification of recycled AMPARs in cultured neurons. The first step labeling was performed as describe in "Two-step labeling of AMPARs or NMDARs in cultured neurons." For the second step, the culture medium was removed and the cells were treated with $1 \mu \mathrm{M} \mathrm{Tz}(\mathrm{Fl})$ for $5 \mathrm{~min}$ in the culture medium at $37^{\circ} \mathrm{C}$. To quench excess $\mathrm{Tz}(\mathrm{Fl}), 1 \mu \mathrm{M}$ TCO-PEG4-COOH in the culture medium was added. After incubation at $37^{\circ} \mathrm{C}$ for $15 \mathrm{~min}$, recycled AMPARs were labeled with $1 \mu \mathrm{M} \mathrm{Tz}(\mathrm{Ax6} 647)$ for $5 \mathrm{~min}$ in PBS. To quench excess Tz(Ax647), $1 \mu \mathrm{M}$ TCO-PEG4-COOH in PBS was added. Cell lysis and western blotting were performed as described in "Two-step labeling of AMPARs or NMDARs in HEK293T cells" using anti-Alexa 647 antibody. Quantification of the band intensity and calculation of the ratio was conducted as described in "Intracellular and surface ratio of labeled AMPARs in HEK293T cells."

Anti-Alexa 647 antibody was prepared from the sera of a rabbit immunized with an antigen which was a conjugate of Alexa 647-NHS and KLH (Sigma), and the antibody was affinity-purified using Alexa 647-conjugated agarose. Alexa 647conjugated agarose was prepared from CarboxyLink Coupling Resin (Thermo Fisher) and Alexa 647 NHS ester (Invitrogen). The anti-sera $(1: 2,000)$ or the purified antibody $(1: 1,000)$ was used for the western blotting.

Preparation of primary hippocampal neuronal culture. Glass bottom dishes (IWAKI) or coverslips (diameter, $13 \mathrm{~mm}$, Matsunami) were coated with poly-Dlysine (Sigma-Aldrich), and washed with sterile $\mathrm{dH}_{2} \mathrm{O}$ three times. Hippocampi from 18-day-old Sprague Dawley rat embryos were aseptically dissected and digested with $0.25 \mathrm{w} / \mathrm{v} \%$ trypsin (Nacalai tesque) for $20 \mathrm{~min}$ at $37^{\circ} \mathrm{C}$. The cells were re-suspended in Neurobasal Plus medium supplemented with $10 \%$ FBS, penicillin $(100$ units $/ \mathrm{ml})$ and streptomycin $(100 \mu \mathrm{g} / \mathrm{ml})$ and filtered by Cell Strainer $(100 \mu \mathrm{m}$, Falcon) and centrifuged at $1,000 \mathrm{rpm}$ for $5 \mathrm{~min}$. The cells were re-suspended in Neurobasal Plus medium supplemented with 2\% of B-27 Plus Supplement, 1.25 mM GlutaMAX I (Invitrogen), penicillin(100 units/ml), and streptomycin $(100 \mu \mathrm{g} /$ $\mathrm{ml}$ ) and plated at a density of $2 \times 10^{4}$ cells on glass coverslips inside 24-well plates (BD Falcon) or glass bottom dishes. Cultures were maintained at $37^{\circ} \mathrm{C}$ in a $95 \%$ air and $5 \% \mathrm{CO}_{2}$ humidified incubator. Culture medium was replaced every 7 days and the neurons were used at 16-18 DIV.

Live cell imaging of AMPARs in cultured neurons. To label endogenous AMPARs, $12 \mu \mathrm{M}$ CAM2(TCO) in $300 \mu \mathrm{l}$ of growth medium with or without 300 $\mu \mathrm{M}$ NBQX was gently added to the hippocampal neurons cultured in $1.5 \mathrm{ml}$ medium on glass bottom dishes to a final concentration of $2 \mu \mathrm{M}$ CAM2(TCO) and $50 \mu \mathrm{M}$ NBQX. After removal of the culture medium, neurons were treated with $100 \mathrm{nM} \mathrm{Tz}(\mathrm{Ax} 488)$ for $5 \mathrm{~min}$ in HBS at room temperature and washed three times with HBS. Confocal live imaging was performed with a confocal microscope.

Immunostaining of cultured neurons after labeling. Primary cultures of hippocampal neurons were labeled by $2 \mu \mathrm{M}$ CAM2(TCO) and followed by $100 \mathrm{nM} \mathrm{Tz}$ (Ax488) as described above. The cells were fixed with 4\% PFA in PBS at room temperature for $30 \mathrm{~min}$ and washed three times with PBS. PFA-fixed cells were permeabilized for $15 \mathrm{~min}$ with PBS containing $0.1 \%$ Triton X-100 at room temperature. The cells were washed three times in PBS and incubated in 10\% normal goat serum for $1 \mathrm{~h}$ at room temperature. After blocking, the cells were incubated overnight at $4{ }^{\circ} \mathrm{C}$ with primary antibodies in PBS containing $1 \%$ normal goat serum. The cells were then washed three times with PBS and incubated for $1 \mathrm{~h}$ at room temperature with secondary antibodies in PBS containing $1 \%$ normal goat serum. The following primary antibodies were used: mouse anti-PSD95 (abcam, ab2723, 1:1,000) or rabbit anti-MAP2 (Millipore, AB5622, 1:1,000). Secondary antibodies were used goat anti-mouse Alexa 647 (abcam, ab150115, 1:1,000) and goat anti-rabbit Alexa633 (Invitrogen, A21070, 1:2,000). Imaging of immunostained hippocampal neurons was performed with a confocal microscope.

Fluorescence lifetime imaging of AMPARs in cultured hippocampal neurons. Primary cultures of hippocampal neurons were labeled by $2 \mu \mathrm{M}$ CAM2(TCO), followed by $100 \mathrm{nM} \mathrm{Tz(ST647)}$ and fixed with 4\% PFA in PBS. The cells were immunolabeled with PSD95 and MAP2 primary antibodies, and stained with Alexa 488 and Alexa405 secondary antibodies, respectively. Confocal and lifetime imaging of immunostained hippocampal neurons was performed by TCS SP8 FALCON (Leica microsystems) equipped with a white light laser and $63 \times, \mathrm{NA}=1.4$ oil-immersion objective. SeTau-647 was imaged using $640 \mathrm{~nm}$ exc. (laser power 100 , emission collected at $653-700 \mathrm{~nm}$, using $0-12.5 \mathrm{~ns}$ time gate).

FLIM images were processed in LAS X 3.5.5 software (Leica microsystems) to fit the lifetime decay curves using an $n$-exponential reconvolution model with the number of components that $\chi^{2}$ value is closest to 1 . In our data, a three-component fit was utilized. The component of the lifetime corresponding to SeTau-647 ( $\tau=$ $2.4 \pm 0.1 \mathrm{~ns}$ ) was used for calculating the intensity of FLIM images. The synaptic or dendric region was selected ROI on PSD95 or MAP2 signals and the background intensities were subtracted by selecting a region of no cells

Statistics and reproducibility. All graphs were generated using Microsoft Excel. All data are expressed as mean \pm s.e.m. We accumulated the data for each condition from at least three independent experiments. We evaluated statistical significance with Student's $t$-test for comparisons between two mean values. A value of $P<0.05$ was considered significant.

Reporting summary. Further information on research design is available in the Nature Research Reporting Summary linked to this article.

\section{Data availability}

The authors declare that the data supporting the findings of this study are available with the paper and its Supplementary information files. The data that support the findings of this study are available from the corresponding author upon reasonable request. Source data are provided with this paper.

Received: 19 May 2020; Accepted: 4 January 2021;

Published online: 05 February 2021

\section{References}

1. Traynelis, S. F. et al. Glutamate receptor ion channels: structure, regulation, and function. Pharmacol. Rev. 62, 405-496 (2010).

2. Bredt, D. S. \& Nicoll, R. A. AMPA receptor trafficking at excitatory synapses. Neuron 40, 361-379 (2003).

3. Diering, G. H. \& Huganir, R. L. The AMPA receptor code of synaptic plasticity. Neuron 100, 314-329 (2018).

4. Paoletti, P., Bellone, C. \& Zhou, Q. NMDA receptor subunit diversity: Impact on receptor properties, synaptic plasticity and disease. Nat. Rev. Neurosci. $\mathbf{1 4}$ 383-400 (2013).

5. Ehlers, M. D. Reinsertion or degradation of AMPA receptors determined by activity-dependent endocytic sorting. Neuron 28, 511-525 (2000).

6. Mammen, A. L., Huganir, R. L. \& O'Brien, R. J. Redistribution and stabilization of cell surface glutamate receptors during synapse formation. J. Neurosci. 17, 7351-7358 (1997).

7. Hanus, C. et al. Unconventional secretory processing diversifies neuronal ion channel properties. eLife 5, e20609 (2016).

8. Kopec, C. D., Li, B., Wei, W., Boehm, J. \& Malinow, R. Glutamate receptor exocytosis and spine enlargement during chemically induced long-term potentiation. J. Neurosci. 26, 2000-2009 (2006).

9. Tanaka, H. \& Hirano, T. Visualization of subunit-specific delivery of glutamate receptors to postsynaptic membrane during hippocampal long-term potentiation. Cell Rep. 1, 291-298 (2012).

10. Keppler, A. et al. A general method for the covalent labeling of fusion proteins with small molecules in vivo. Nat. Biotechnol. 21, 86-89 (2003).

11. Los, G. V. et al. HaloTag: A novel protein labeling technology for cell imaging and protein analysis. ACS Chem. Biol. 3, 373-382 (2008).

12. Hirayama, S., Hori, Y., Benedek, Z., Suzuki, T. \& Kikuchi, K. Fluorogenic probes reveal a role of GLUT4 N-glycosylation in intracellular trafficking. Nat. Chem. Biol. 12, 853-859 (2016).

13. Griffin, B. A., Adams, S. R. \& Tsien, R. Y. Specific covalent labeling of recombinant protein molecules inside live cells. Science 281, 269-272 (1998).

14. Chen, I., Howarth, M., Lin, W. \& Ting, A. Y. Site-specific labeling of cell surface proteins with biophysical probes using biotin ligase. Nat. Methods 2, 99-104 (2005)

15. Nonaka, H., Fujishima, S. H., Uchinomiya, S. H., Ojida, A. \& Hamachi, I. Selective covalent labeling of tag-fused GPCR proteins on live cell surface with a synthetic probe for their functional analysis. J. Am. Chem. Soc. 132, 9301-9309 (2010).

16. Neubert, F. et al. Bioorthogonal click chemistry enables site-specific fluorescence labeling of functional NMDA receptors for super-resolution imaging. Angew. Chem. Int. Ed. 57, 16364-16369 (2018).

17. Tsai, Y. H., Essig, S., James, J. R., Lang, K. \& Chin, J. W. Selective, rapid and optically switchable regulation of protein function in live mammalian cells. Nat. Chem. 7, 554-561 (2015).

18. Humeau, Y. \& Choquet, D. The next generation of approaches to investigate the link between synaptic plasticity and learning. Nat. Neurosci. 22, 1536-1543 (2019).

19. Fleming, J. J. \& England, P. M. AMPA receptors and synaptic plasticity: a chemist's perspective. Nat. Chem. Biol. 6, 89-97 (2010).

20. Cravatt, B. F., Wright, A. T. \& Kozarich, J. W. Activity-based protein profiling: from enzyme chemistry to proteomic chemistry. Annu. Rev. Biochem. 77, 383-414 (2008). 
21. Fehrentz, T., Schönberger, M. \& Trauner, D. Optochemical genetics. Angew. Chem. Int. Ed. 50, 12156-12182 (2011).

22. Tsukiji, S., Miyagawa, M., Takaoka, Y., Tamura, T. \& Hamachi, I. Liganddirected tosyl chemistry for protein labeling in vivo. Nat. Chem. Biol. 5, 341-343 (2009).

23. Tamura, T. et al. Rapid labelling and covalent inhibition of intracellular native proteins using ligand-directed $\mathrm{N}$-acyl-N-alkyl sulfonamide. Nat. Commun. 9, 1870 (2018).

24. Fujishima, S. H., Yasui, R., Miki, T., Ojida, A. \& Hamachi, I. Ligand-directed acyl imidazole chemistry for labeling of membrane-bound proteins on live cells. J. Am. Chem. Soc. 134, 3961-3964 (2012).

25. Miki, T. et al. LDAI-based chemical labeling of intact membrane proteins and its pulse-chase analysis under live cell conditions. Chem. Biol. 21, 1013-1022 (2014).

26. Wakayama, S. et al. Chemical labelling for visualizing native AMPA receptors in live neurons. Nat. Commun. 8, 14850 (2017).

27. Sekine-Aizawa, Y. \& Huganir, R. L. Imaging of receptor trafficking by using $\alpha$ bungarotoxin-binding-site-tagged receptors. Proc. Natl Acad. Sci. USA 101, 17114-17119 (2004).

28. Kanda, H. \& Gu, J. G. Effects of cold temperatures on the excitability of rat trigeminal ganglion neurons that are not for cold sensing. J. Neurochem. 141, 532-543 (2017).

29. Tabernero, A. et al. Albumin promotes neuronal survival by increasing the synthesis and release of glutamate. J. Neurochem. 81, 881-891 (2002).

30. Atabay, C., Cagnoli, C. M., Kharlamov, E., Ikonomovic, M. D. \& Manev, H. Removal of serum from primary cultures of cerebellar granule neurons induces oxidative stress and DNA fragmentation: Protection with antioxidants and glutamate receptor antagonists. J. Neurosci. Res. 43, 465-475 (1996).

31. Blackman, M. L., Royzen, M. \& Fox, J. M. Tetrazine ligation: fast bioconjugation based on inverse-electron-demand Diels-Alder reactivity. J. Am. Chem. Soc. 130, 13518-13519 (2008).

32. Devaraj, N. K., Weissleder, R. \& Hilderbrand, S. A. Tetrazine-based cycloadditions: application to pretargeted live cell imaging. Bioconjug. Chem. 19, 2297-2299 (2008).

33. Oliveira, B. L., Guo, Z. \& Bernardes, G. J. L. Inverse electron demand DielsAlder reactions in chemical biology. Chem. Soc. Rev. 46, 4895-4950 (2017).

34. Ritchie, T. J. \& Macdonald, S. J. F. The impact of aromatic ring count on compound developability - are too many aromatic rings a liability in drug design? Drug Discov. Today 14, 1011-1020 (2009).

35. Devaraj, N. K., Hilderbrand, S., Upadhyay, R., Mazitschek, R. \& Weissleder, R. Bioorthogonal turn-on probes for imaging small molecules inside living cells. Angew. Chem. Int. Ed. 49, 2869-2872 (2010).

36. Liu, D. S. et al. Diels-alder cycloaddition for fluorophore targeting to specific proteins inside living cells. J. Am. Chem. Soc. 134, $792-795$ (2012)

37. Beliu, G. et al. Bioorthogonal labeling with tetrazine-dyes for super-resolution microscopy. Commun. Biol. 2, 261 (2019).

38. Takeuchi, Y., Morise, J., Morita, I., Takematsu, H. \& Oka, S. Role of site-specific $\mathrm{N}$-glycans expressed on GluA2 in the regulation of cell surface expression of AMPA-type glutamate receptors. PLoS One 10, e0135644 (2015).

39. Podgorski, K., Terpetschnig, E., Klochko, O. P., Obukhova, O. M. \& Haas, K. Ultra-bright and -stable red and near-infrared squaraine fluorophores for in vivo two-photon imaging. PLoS ONE 7, e51980 (2012).

40. Tsunoyama, T. A. et al. Super-long single-molecule tracking reveals dynamicanchorage-induced integrin function. Nat. Chem. Biol. 14, 497-506 (2018).

41. Janssens, N. \& Lesage, A. S. J. Glutamate receptor subunit expression in primary neuronal and secondary glial cultures. J. Neurochem. 77, 1457-1474 (2001).

42. Ashby, M. C., Maier, S. R., Nishimune, A. \& Henley, J. M. Lateral diffusion drives constitutive exchange of AMPA receptors at dendritic spines and is regulated by spine morphology. J. Neurosci. 26, 7046-7055 (2006).

43. O'Brien, R. J. et al. Activity-dependent modulation of synaptic AMPA receptor accumulation. Neuron 21, 1067-1078 (1998).

44. Archibald, K., Perry, M. J., Molnár, E. \& Henley, J. M. Surface expression and metabolic half-life of AMPA receptors in cultured rat cerebellar granule cells. Neuropharmacol 37, 1345-1353 (1998).

45. Tomita, S., Fukata, M., Nicoll, R. A. \& Bredt, D. S. Dynamic interaction of stargazin-like TARPs with cycling AMPA receptors at synapses. Science 303, 1508-1511 (2004).

46. Kinney, W. A. et al. Design and synthesis of [2-(8,9-dioxo-2,6-diazabicyclo [5.2.0]non-1(7)-en-2-yl)-ethyl]phosphonic acid (EAA-090), a potent $\mathrm{N}$ methyl-D-asparate antagonist, via the use of 3-cyclobutene-1,2-dione as an achiral a-amino acid bioisostere. J. Med. Chem. 41, 236-246 (1998).

47. Cohen, L. D. et al. Metabolic turnover of synaptic proteins: kinetics, interdependencies and implications for synaptic maintenance. PLOS ONE $\mathbf{8}$ e63191 (2013).
48. Miyazaki, T. et al. Visualization of AMPA receptors in living human brain with positron emission tomography. Nat. Med. 26, 281-288 (2020).

49. Howard, M. A., Elias, G. M., Elias, L. A. B., Swat, W. \& Nicoll, R. A. The role of SAP97 in synaptic glutamate receptor dynamics. Proc. Natl Acad. Sci. USA 107, 3805-3810 (2010).

50. Wyszynski, M. et al. Association of AMPA receptors with a subset of glutamate receptor-interacting protein in vivo. J. Neurosci. 19, 6528-6537 (1999).

51. Lisman, J., Yasuda, R. \& Raghavachari, S. Mechanisms of CaMKII action in long-term potentiation. Nat. Rev. Neurosci. 13, 169-182 (2012).

52. Hell, J. W. How $\mathrm{Ca}^{2+}$-permeable AMPA receptors, the kinase PKA, and the phosphatase PP2B are intertwined in synaptic LTP and LTD. Sci. Signal. 9, pe2 (2016).

53. Lin, A. et al. Nedd4-mediated AMPA receptor ubiquitination regulates receptor turnover and trafficking. J. Neurochem. 119, 27-39 (2011).

54. Huo, Y. et al. The deubiquitinating enzyme USP46 regulates AMPA receptor ubiquitination and trafficking. J. Neurochem. 134, 1067-1080 (2015).

55. Matsuda, K., Kamiya, Y., Matsuda, S. \& Yuzaki, M. Cloning and characterization of a novel NMDA receptor subunit NR3B: a dominant subunit that reduces calcium permeability. Mol. Brain Res. 100, 43-52 (2002).

\section{Acknowledgements}

The authors thank Mr. Hideyuki Yamaguchi (Leica Microsystems) for technical supports in FLIM imaging and Dr. Bronwen Gardner (Edanz Group) for editing a draft of this manuscript. This work was funded by Grants-in-Aid for Scientific Research (KAKENHI) (Grant Number 18J22952 to K.O., 17H06348 to I.H., 16H03290, 19H05778, and 20H02877 to S.K.), Daiichi Sankyo Foundation of Life Science, the Takeda Science Foundation, and the Mochida Memorial Foundation for Medical and Pharmaceutical Research to S.K., and supported by JST CREST (JPMJCR1854) to M.Y. and JST ERATO Grant Number JPMJER1802 to I.H.

\section{Author contributions}

S.K. and I.H. initiated and designed the project. K.O., K.Shiraiwa, K.Soga, T.D., M.T. and K.K. performed synthesis and chemical labeling in HEK293T cells. K.O., K.Shiraiwa, M.Y. and S.K. performed chemical labeling in cultured neurons. K.O. and S.K. wrote the manuscript. All authors discussed and commented on the manuscript.

\section{Competing interests}

The authors declare no competing interests.

\section{Additional information}

Supplementary information The online version contains supplementary material available at https://doi.org/10.1038/s41467-021-21082-x.

Correspondence and requests for materials should be addressed to I.H. or S.K.

Peer review information Nature Communications thanks the anonymous reviewers for their contribution to the peer review of this work. Peer reviewer reports are available.

Reprints and permission information is available at http://www.nature.com/reprints

Publisher's note Springer Nature remains neutral with regard to jurisdictional claims in published maps and institutional affiliations.

Open Access This article is licensed under a Creative Commons Attribution 4.0 International License, which permits use, sharing, adaptation, distribution and reproduction in any medium or format, as long as you give appropriate credit to the original author(s) and the source, provide a link to the Creative Commons license, and indicate if changes were made. The images or other third party material in this article are included in the article's Creative Commons license, unless indicated otherwise in a credit line to the material. If material is not included in the article's Creative Commons license and your intended use is not permitted by statutory regulation or exceeds the permitted use, you will need to obtain permission directly from the copyright holder. To view a copy of this license, visit http://creativecommons.org/ licenses/by/4.0/

(C) The Author(s) 2021 